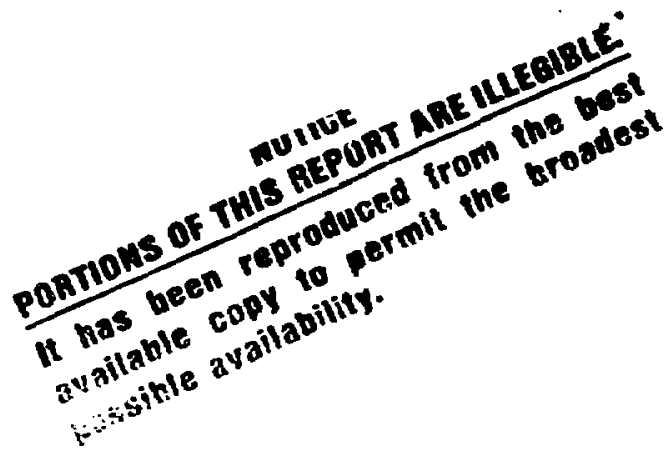

$\mathrm{LA}--10235-\mathrm{MS}$

DE85 007378

\title{
Documentation for TRACE: An Interactive Beam-Transport Code
}

\author{
K. R. Crandall \\ D. P. Rusthoi
}

\section{DISCLAIMER}

This report was prepared as an account of work uponsored by an agency of the United Statea Government. Neither the United Statea Government nor any agency thereof, nor any of their employees, makes any warranty, express or implied, or asumes any legal liability or responsibility for the sccuracy, completeneas, or uefulneas of any information, apparatus, product, or process disclowed, or represents that its use would not infringe privately owned rights. Reference herein to any specific cornmercial product, proceas, or service by trade name, trademark, manufacturer, or otherwive does not necesurily constitute or imply its endonement, recommendation, or favoring by the United States Government or any egency thereof. The views and opinions of authors expreaced herein do not necesarily state or reflect those of the United States Government or any ageacy theseof. 
CONTENTS

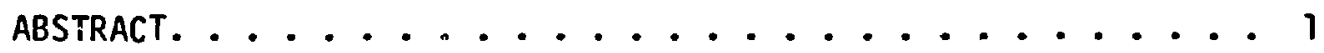

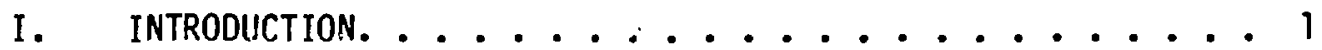

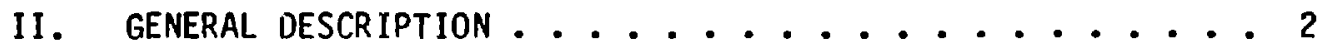

A. Equations of Motion ........... 2

B. Matrix Formulation........... 3

C. Ellipse Transformations .......... 3

D. Computational Procedure ........... 4

E. Emittance ............... 4

III. TRANSPORT-SYSTEM SPECIFICATION. .......... 5

A. Transport Elements. ........... 5

B. Description of Element Parameters and

External-force Constants ......... 5

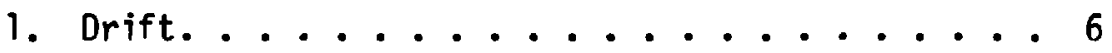

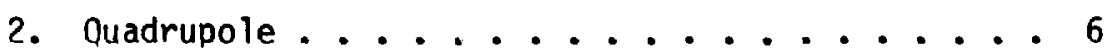

3. Symmetric Doublet. .......... 7

4. Bending Magnet ............ 7

5. Symmetric Triplet............ 9

6. Buncher/Debuncher Simulator. ....... 10

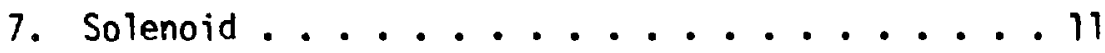

8. Tapered $\mathrm{rf}$ Quadrupole........... 11

9. The $r f$ Gap ............... 13

10. Thin Lens............... 14

11. Accelerator Tank ............. 14

12. Accelerator Column ............ 17

13. Current Change ........... 18

14. Horizontal R-Matrix. ......... 18

15. Vertical R-Matrix........... 19

16. Permanent-Magnet Ouadrupole. . . . . . 19 


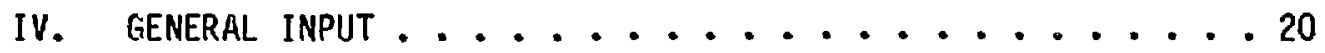

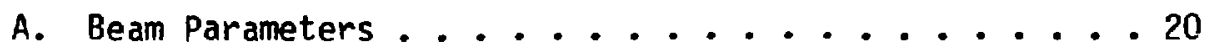

B. Fitting Parameters. ............ 21

C. Graphics Display. ............. 24

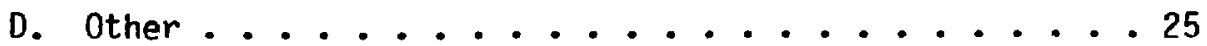

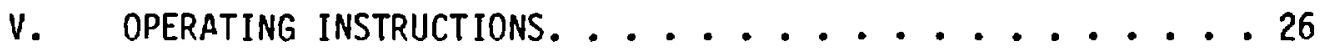

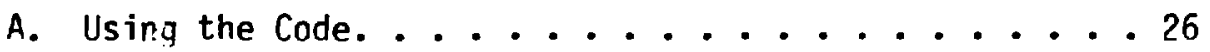

B. Description of Interactive Commands ...... 28

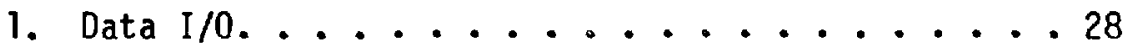

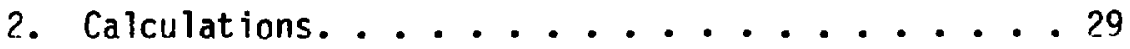

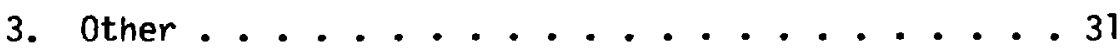

VI. MATCHING CAPABILITIES ......................

A. Types of Matching ............ 33

B. Definition of Mismatch Factor ........ 37

APPENDIX A. EMITTANCE CALCULATIONS THROUGH THE BUNCHER . . . 39 APPENDIX B. ACCEL.ERATOR COLUMN MODEL . . . . . . . . 42 APPENDIX C. PERMANENT-MAGNET-QUADRUPOLE FIELD FORMULAE . . . . 45 APPENDIX D. SPACE-CHARGE CALCULATIONS. . . . . . . . 48 APPENDIX E. SAMPLE OUTPUT. . . . ........ 50 APPENDIX F. HELP PACKAGE .............. 57 APPENDIX G. GRAPHICS PACKAGE ............. 61

REFERENCES.................... 62 
DOCUMENTATION FOR TRACE: AN INTERACTIVE BEAM-TRANSPORT CODE

by

K. R. Crandall and D. P. Rusthoi

\begin{abstract}
TRACE is an interactive, first-order, beam-dynamics computer program. TRACE includes space-charge forces and mathematical models for a number of beamline elements not commonly found in beam-transport. codes, such as permanent-magnet quadrupoles, if quadrupoles, if gaps, accelerator columns, and accelerator tanks. TRACE provides an immediate graphic display of calculative results, has a powerful and easy-to-use command procedure, includes eight different types of beam-matching or -fitting capabilities, and contains; its own internal HELP package. This report describes the models ard equations used for each of the transport elements, the fititing procedures, and the space-charge/emittance calculations, and provides detailed instruction for using the code.
\end{abstract}

\title{
I. INTRODUCTION
}

TRACE is an interactive, first-order, envelope-tracing, beam-dynamics program with space charge. It uses the Courant-Snyder notation $(\alpha, \beta, \gamma)$, does dynamics calculations using two 2 by 2 matrices, and superimposes defocusing forces proportional to beam size when space charge is involved.

The original version of TRACE was written in $1973^{1}$ and was developed for use on the controls computer of LAMPF. That version facilitated beam-transport calculations, starting with measured properties of the LAMPF beam, and served as an aid in tuning the beam transport. A modified version was written for the controls computer of the CERN linac in $1977 ;^{2}$ in 1979, the CERN version was adapted to the Pion Generator for Medical Irradiations (PIGMI) controls system and was used in tuning the transport for the test of the radio-frequency quadrupole (RFQ) linac. Because of its usefulness as a design tool as well as a 
tuning aid, TRACE was expanded and adapted for use on the CDC computers 16600 and 7600 ) at the Los Alamos National Laboratory central computing facility, and for use on the VAX-11/750. This report details the 1984 version of TRACE and can also serve as a user's manual.

TRACE has some unique features as well as representations of a number of transport elements not commonly found in other beam-transport codes, including the permanent-magnet quadrupole (PMQ), the RFO, the rf gap, the buncher/ debuncher simulator, the accelerator column, and the accelerator tank. The code has a number of fitting capabilities, allowing almost any element parameter in the beamline to be varied, including space charge. TRACE calculations provide immediate graphic display, including the beam envelopes and the phasespace ellipses in the transverse dimensions. The program is easy to use and contains its own HELP package that lists the instructions necessary for input, calculations, and graphic output.

\section{GENERAL DESCRIPTION}

\section{A. Equations of Motion}

TRACE, being a first-order code, assumes the equations of motion can be expressed as

$$
x^{\prime \prime}+k_{x}{ }^{2} x=0 \quad \text { and } \quad y^{\prime \prime}+k_{y}{ }^{2} y=0 \text {, }
$$

where the prime denotes differentation with respect to distance along the central trajectory, and where the constants $k_{x}{ }^{2}$ and $k_{y}{ }^{2}$ include the space-charge forces as well as the external forces (for example, $k_{x}{ }^{2}=k_{e}, x^{2}+k_{s,} x^{2}$ where $k_{e}, x^{2}$ is the $x$-component of the force constant for external fieids and $k_{s, x^{2}}$ is the $x$-component of the force constant for space-charge forces). For the space-charge forces to be linear, one must postulate a beam having a uniform charge distribution. Although real life is rarely so accommodating, it has been shown that for distributions having ellipsoidal symetry, the evolution of the rms beam envelope depends almost exclusively on the linearized part of the self-forces." Consequently, for calculational purposes the "real bean!" may be replaced by an "equivalent beam" having the same rms properties, but of uniform distribution. 


\section{B. Matrix Formulation}

The above differential equations have simple solutions. If one knows the $x, x^{\prime}, y, y^{\prime}$ coordinates of a particle at some longitudinal position $z$, then the coordinates at $z+\Delta z$ are given by

$$
\vec{x}(z+\Delta z)=R_{x} \vec{X}(z) \quad \text { and } \quad \vec{y}(z+\Delta z)=R_{y} \vec{y}(z) \text {, }
$$

where $\vec{x}$ and $\vec{y}$ denote the column vectors $\left(\begin{array}{l}x \\ x^{\prime}\end{array}\right)$ and $\left(\begin{array}{l}y \\ y^{\prime}\end{array}\right)$ and $R_{x}$ and $R_{y}$ are the $x$ - and $y$-transfer matrices that depend on the constants $k_{x}{ }^{2}$ and $k_{y}{ }^{2}$.

$$
\begin{aligned}
& R=\left[\begin{array}{rr}
\cos (k \Delta z) & \frac{l}{k} \sin (k \Delta z) \\
-k \sin (k \Delta z) & \cos (k \Delta z)
\end{array}\right] \text { when } k^{2} \geq 0, \text { and } \\
& R=\left[\begin{array}{cc}
\cosh (k \Delta z) & \frac{l}{k} \sinh (k \Delta z) \\
k \sinh (k \Delta z) & \cosh (k \Delta z)
\end{array}\right] \text { when } k^{2}<0 .
\end{aligned}
$$

In this context, $k=\sqrt{\left|k^{2}\right|}$ and this equivalence applies throughout this report.

\section{Ellipse Transformations}

TRACE is not a particle-tracing code; rather, it calculates the behavior of the "beam ellipses." In heam-dynamics work, it is common practice to express the $x-x$ ' beam ellipse as

$$
\gamma x^{2}+2 \alpha x x^{\prime}+B x^{\prime 2}=E / \pi,
$$

where $\alpha, B$, and $\gamma$ are the Twiss (or Courant-Snyder) parameters satisfying the relation $B \gamma-\alpha^{2}=1$, and $E$ is the emittance, or the area of the phase-space ellipse. A similar expression defines the $y-y^{\prime}$ beam ellipse. The so-called "beam matrix," often used in beam-transport calculations, is defined as

$$
\sigma=\frac{E}{\pi}\left[\begin{array}{cc}
\beta & -\alpha \\
-\alpha & \gamma
\end{array}\right] .
$$


If the beam matrices $\sigma_{x}$ and $\sigma_{y}$ are known at location $z$, and if the transfer matrices $R_{x}$ and $R_{y}$ are known, the beam matrices at $z+\Delta z$ are given by

$$
\sigma_{x}(z+\Delta z)=R_{x} \sigma_{x}(z) R_{x}^{\top}, \quad \text { and } \quad \sigma_{y}(z+\Delta z)=R_{y} \sigma_{y}(z) R_{y}^{\top}
$$

where $R^{T}$ denotes the transpose of $R$.

D. Computational Procedure

The computational procedure within the program is as follows:

- Initial beam matrices are constructed from the specified Twiss parameters ;

- Each transport element is divided into an integer number of segments, where the inteqer is determined by a specified maximum step size;

- if any permanent-magnet fringe fields are extant at the point of calculation, they are calculated for inclusion in the transfer matrix (superpositioning);

- The $x$ - and $y$-transfer matrices are calculated for one computational step, and if $k_{x}{ }^{2}$ and $k_{y}{ }^{2}$ are not constant for that step (as will be the case for some elements and when spare-charge forces are involved), average values are used; and

- The beam matrices are calculated at the end of the computational step.

New transfer matrices are constructed and beam matrices calculated at each step until the beam has been transported through the specified portion of the transport system.

Normally, at the beginning of the calculation, the initial beam ellipses are displayed at the graphics terminal. At each step, the maximum extent of the beam in the transverse plane is calculated and displayed, so that one can see the beam profiles. At the end, the final beam ellipses are displayed.

E. Emittance

When using TRACE to solve problems in which space-charge forces are included (the common situation), the values specified for the emittance in the $x-x^{\prime}$ and $y-y^{\prime}$ phase $n$ lanes should be four times the value of the rms emittance in the respective phase $p l a n e$. The rms emittance is defined as the area of the ellipse that passes through the rms properties of the beam. That is 


$$
E_{x, r m s}=\pi \sqrt{\overline{x^{2}\left(x^{\prime}\right)^{2}}-(\bar{x} \bar{x})^{2}} \text {, }
$$

where the bars denote averages. The rms properties of a beam are usually among the easiest quantities to measure. The multiplication factor of 4 is used because a uniformly distributed ellipse has an area four times as large as its rms area.

Also in this regard, the "edge" of the beam (or beam profile), as displayed by TRACE, occurs at twice the rms value:

$$
x_{\max }=2 \sqrt{x^{2}}
$$

The edge of a real beam would normally extend beyond this value.

\section{TRANSPORT-SYSTEM SPECIFICATION}

\section{A. Transport Elements}

The transport elements are defined by a "type-code" and by five or fewer parameters, except for a bending magnet when nine parameters can be specified. A transport system consists of a sequence of 50 or fewer elements. The typecode for each element is stored in the array NT( $n)$ and the parameters in array $A(1, n)$ through $A(5, n)$ where " $n$ " is the sequential number of a particular element in the transport system. The 16 elements recognized by TRACE are given in Table I along with their type codes and symbols for associated parameters.

\section{B. Description of Element Parameters and External-Force Constants}

Unless otherwise specified, all conventions assume a positive beam, all lengths are in millimeters [mm], all magnetic field gradients are in teslas/ meter $[\mathrm{T} / \mathrm{m}]$, and all angles are in degrees [deg]. To specify an element in the transport system, both the element type-code NT discussed above: and the element parameters A discussed below, must be entered. For some elements, further discussion of the models and equations are included in the appendixes: Appendix A details the emittance calculations through the buncher; Appendix $B$ describes the accelerator column model and the approximations used; Appendix $C$ gives the formulae for calculating the fringe fields for permanent-magnet quadrupoles; and Appendix $D$ describes the space-charge calculations. 


\section{TABLE I}

\section{LIST OF ELEMENTS}

\section{Element}

Drift

Quadrupole

Symmetric doublet

Bending magnet (continuation)

Symmetric triplet

Buncher/debuncher

Solenoid

Tapered rf quadrupole

The rf gap

Thin lens

Accelerator tank

Accelerator column

Current change

R-matrix (horiz.)

R-matrix (vertical)

Permanent-magnet quad
Typo

Code Parameters

$1 l$

$2 \quad B^{\prime}, l$

$3 \quad \mathrm{~B}^{\prime}, l, \mathrm{~s}$

$4 \quad \alpha, \rho, \beta_{1}, \beta_{2}, g$

$0, \mathbf{n}, b_{1} k_{1}$

$5 \quad B_{0}^{\prime} l_{0}, l_{0}, B_{i}^{\prime}, l_{i}$

$6 \Delta \phi_{\mathrm{i}}, \Delta \phi_{\mathrm{f}}, \mathrm{d}, \Delta \mathrm{W}, \mathrm{V}_{\mathrm{r}}$

7 Bs, $l$

$8 B_{1}, B_{2}, n_{\beta \lambda}, n_{3}, \phi$

9 EoT, $\Delta W, \phi_{3}$

$10 \quad 1 / f$

$11 l_{t}, \phi_{s}, n_{c}, W_{i}, W_{0}$

$12 \quad r_{a}, l, \Delta W, n_{s}, I$

$13 \Delta I$

$14 R_{11}, R_{12}, R_{21}, R_{22}$

$15 R_{33}, R_{34}, R_{43}, R_{44}$

$16 \quad \mathrm{~B}^{\prime}, l_{\mathrm{p}}, \mathrm{r}_{\mathrm{i}}, \mathrm{r}_{\mathrm{o}}, \mathrm{st}$

1. Drift

$A(1, n)=$ drift. length $\&[\mathrm{~mm}]$

The only parameter to specify is the lenath of the drift $\ell$. Both external force constants are zero:

$k_{e, x}{ }^{?}=k_{e, y^{2}}=0$.

2. Quadrupole

$A(?, n)=$ field gradient $B^{\prime}[T / m]$

$A(2, n)=$ effective length $\ell[\mathrm{mm}]$ 
The two parameters to be specified are the quadrupole gradient $B^{\prime}$ and the effective length $\ell$. The force constants are

$$
k_{e, x^{2}}=-k_{e, y^{2}}=B^{\prime} / B \rho,
$$

where $B \rho$ is the magnetic rigidity of the particle defined by

$$
B \rho=\frac{m_{0} c R_{y}}{q e} .
$$

In this expression, $m_{0}$ is the rest mass, $c$ is the velocity of light, $q$ is the charge state, $e$ is the magniture of the charge on an electron, and $\beta$ and $\gamma$ are the standard relativistic parameters. For protons,

$$
B p=3.1295 \text { By tesla-meters. }
$$

For positive beams, a positive value for the quadrupole gradient B' focuses in the $x-p l$ ane and defocuses in the $y$-plane.

3. Symmetric Doublet

$$
\begin{aligned}
& A(1, n)=\text { field gradient } B^{\prime}\lceil T / m\rceil \\
& A(2, n)=\text { effective length } \ell\lceil\mathrm{mm}] \\
& A(3, n)=\text { drift distance between quadrupoles } s[\mathrm{~mm}]
\end{aligned}
$$

A symmetric douhlet consists of two identical quadrupoles each having an effective length $\&$ separated by a drift length $s$. The parameter $B^{\prime}$ is the gradient in the upstream quadrupole (the input value) and $-B^{\prime}$ ' is the gradient in the downstream quadrupole. The force constants are defined in the same manner as for the drift and quadrupole elements.

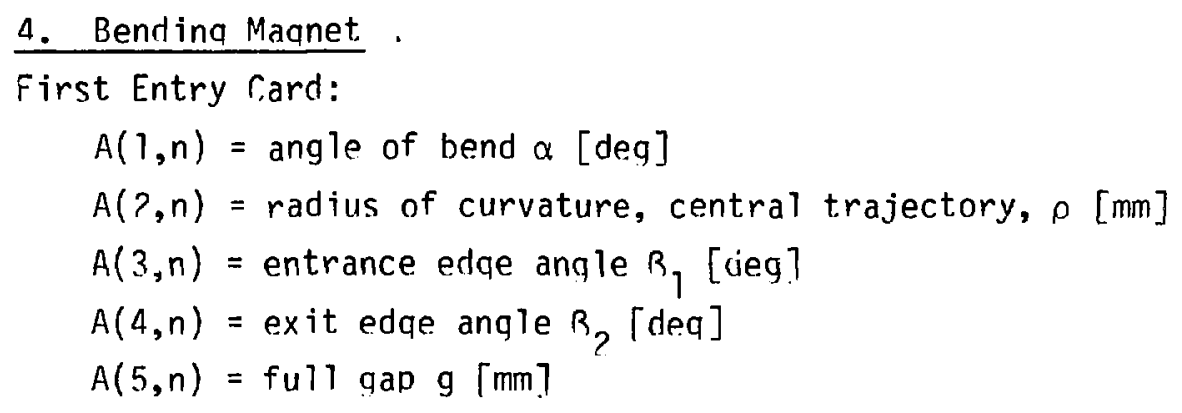


Second Entry Card (continuation):

$$
\begin{aligned}
& A(1, n)=0 \text { (zero-degree bend-angle flags entry as continuation) } \\
& A(2, n)=\text { field gradient } n \text { (dimensionless, } 0<n<1) \\
& A(3, n)=\text { bend-plane flag } b(0=\text { horiz., } 1=\text { vert.) } \\
& A(4, n)=\text { fringe field factor } k_{1} \text { (default }=0.45 \text { ) }
\end{aligned}
$$

The bending magnet requires at least two input parameters, namely, the angle of bend $\alpha$ and the radius of curvature $\rho$. There can be as many as eight input parameters to characterize the element. The model is a wedge-type bending magnet where the central trajectory enters and exits perpendicularly to the pole-face boundaries unless edge angles of the entrance and/or exit are specified (the default is $0^{\circ}$ ). For positive bends ( $\alpha$ and $\rho>0$ ), a positive edge angle $B$ will focus vertically. Negative edge angles focus horizontally. The sign conventions are the same as those used in the TRANSPORT code as illustrated in Fig. 1. The effects of fringing fields are ignered unless an entry is made for the gap. The qap is considered vertical (and bend plane horizontal) unless otherwise indicated on the continuation card. The continuation card is another bending-magnet entry (another element but with no length), having a zero-degree bend $[A(1, n)=0]$. The zero-degree bend flags the entry as continuation data for the bending magnet immediately preceding or following it. ${ }^{*}$ The additional parameters are the field gradient, $n$, a dimensionless quantity $[0<n<1]$, the bend.-plane flag b $(0=$ horizontal, 1 = vertical $)$, and the fringe-field factor $k_{7}$. The fringe-field factor is an integral related to the extent of the fringing field. The default for $k_{1}$ is 0.45 , which is typical for a squareedged, nonsaturating magnet. The default value is used if no entry or a zero is entered for $k_{1}$.

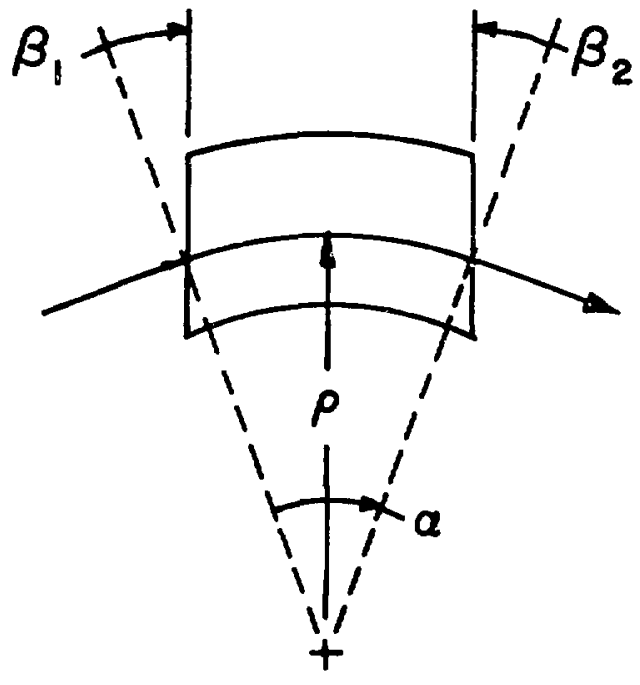

Fig. 1. Wedge-type bending magnet showing TRACE sign convention for $\alpha, \rho$, $B_{1}$, and $B_{2}$. All values as shown are positive.

\footnotetext{
*Note that if two bending magnets with different continuation cards are used adjacent to each other, a zero-length drift should be placed between them to ensure that the appropriate continuation card is used.
} 
The edge-angle transformation in the plane of the bend (assumed horizontal) is

$$
R_{X}=\left[\begin{array}{cc}
1 & 0 \\
\frac{\tan B}{\rho} & 1
\end{array}\right]
$$

and in the other plane (assumed vertical) is

$$
R_{y}=\left[\begin{array}{cc}
1 & 0 \\
\frac{-1}{\rho} \tan (B-\psi) & 1
\end{array}\right] \text {, }
$$

where

$$
\psi=k_{1}\left(\frac{g}{\rho}\right)\left[\frac{1+\sin ^{2} \beta}{\cos B}\right]\left[1-2.8 k_{1}\left(\frac{g}{\rho}\right) \tan B\right] .
$$

Inside the bend, the force constants are

$$
\begin{aligned}
& k_{e, x^{2}}=(1-n) / \rho^{2}, \text { and } \\
& k_{e, y^{2}}=n / \rho^{2} .
\end{aligned}
$$

5. Symmetric Triplet

$$
\begin{aligned}
& A(1, n)=\text { field gradient (outer quadrupoles) } B^{\prime}{ }_{0}[T / m] \\
& A(2, n)=\text { effective length (outer quadrupoles) } \ell_{0}[\mathrm{~mm}] \\
& A(3, n)=\text { drift distance (hetween inner and an outer quadrupole) } \mathrm{s}[\mathrm{mm}] \\
& A(4, n)=\text { field gradient (inner quadrupole) } B^{\prime}{ }_{i}[T / \mathrm{m}] \\
& A(5, n)=\text { effective length (inner quadrupole) } \ell_{i}[\mathrm{~mm}]
\end{aligned}
$$

A symmetric triplet consists of two identical outer quadrupoles, each having a field aradient $B^{\prime}{ }_{0}$ and effective length $\ell_{0}$. The distance between the inner and outer quadrupoles is $s$, and $B^{\prime}{ }_{i}$ and $\ell_{i}$ are the gradient and effective length of the inner quatrupole. The force constants are defined in the same manner as for the orift and quadrupole elements. 


$$
\begin{aligned}
& \text { 6. Buncher/Debuncher Simulator } \\
& A(1, n)= \text { initial half-phase spread } \Delta \phi_{j} \text { [deg] } \\
& A(2, n)= \text { final half-phase spread } \Delta \phi_{f}[\text { deq] } \\
& A(3, n)= \text { distance, from buncher for complete b'inching or } \\
& \text { debunching, } d[\mathrm{~mm}] \\
& A(4, n)= \text { maximum energy gain at fundamental frequency } \Delta W[\mathrm{keV}] \\
& A(5, n)= \text { voltage ratio } V_{r} \text { (harmonic buncher voltage/fundamental } \\
& \text { buncher voltage) }
\end{aligned}
$$

The buncher/debuncher simulator is useful for calculating the changing transverse space-charge forces on a bunched beam. This calculation is done by multiplying the unbunched beam forces in TRACE by a factor based on the fraction of beam contained in a changing phase spread as a function of distance. These forces can be caiculated from the first three input parameters: (1) the initial half-phase spread $\Delta \phi_{j}$, at the location of the buncher; (2) the final half-phase spread $\Delta \phi_{f}$, at a distance $d$ from the buncher, where bunching or debunching is terminated; and (3) the predetermined distance $d$, betweer, the buncher and the end point for bunching. These first three parameters specify that the portion of beam within a phase spread of $2 \Delta \phi_{i}$ at the buncher wili be bunched (or debunched) to lie within a phase spread of $2 \Delta \phi_{f}$ after a distance $d$. At a distance $z$, downstream of the buncher (where $0 \leq z \leq d$ ), the phase spread containing the beam initially contained within $2 \Delta \phi_{j}$ is

$$
\Delta \phi(z)=2 \Delta \phi_{i}+\frac{2 z}{d}\left(\Delta \phi_{f}-\Delta \phi_{i}\right)
$$

The space-charge force is calculated by multiplying the unbunched beam force by the factor

$$
F(z)=2 \Delta \phi_{j} / \Delta \phi(z) \text {. }
$$

When used in the bunching mode (that is, $\Delta \phi_{i}>\Delta \phi_{f}$ ), and for distances $z>d$, it is assumed that the beam starts debunching at the same rate as it was bunching. The phase spread is calculated as above except that $z$ is replaced by $z^{\prime}$ : $z^{\prime}=2 d-z$.

The force is that of a long, uniformly charged cylinder whose effective current is $F \cdot I$, where $I$ is the current in the unbunched beam.

The last two parameters in the buncher element are only used in calculating the change in the transverse emittance when the beam passes through a 
buncher (see Appendix A). Note that because the buncher frequency is required, a value for wavelength must be entered (a general input parameter, see Sec. IV).

Because of the approximate way that the bunching effect is treated, there can be only one buncher in a transport system. If the transport system contains a buncher, then (any time the beam is downstream of the buncher) the effective value is used for the current. Any current-change elements (NT = 13) downstream of the buncher are ignored. However, when the beam is upstream of the buncher, the standard beam current is used, and current-change cards are valid.

7. Solenoid

$$
\begin{aligned}
& A(1, n)=\text { field strength } B_{S}[G] \\
& A(\hat{c}, n)=\text { effective length } \&[\mathrm{~mm}]
\end{aligned}
$$

The solenoid formulation in TRACE is valid only for axially symmetric beams because the focusing forces are calculated in a rotating frame of reference, the so-called Larmor frame. The parameters are $B_{s}$, the axial magnetic flux density in gauss, and the effective length $\ell$. The force constants are

$$
\begin{aligned}
& k_{e}, x^{2}=k_{e, y^{2}}=\left(B_{s} / 2 B_{\rho}\right)^{2} \\
& \text { 8. Tapered } r f \text { Ouadrupole } \\
& \hline A(1, n)= \text { amplitude of focusing field, at upstream end, } B_{1} \\
& A(2, n)= \text { amplitude of focusing field, at downstream end, } B_{2} \\
& A(3, n)= \text { length of element, divided by beta-lambda or number } \\
& \text { of beta-lambdas, } n_{B \lambda} \\
& A(4, n)= \text { number of steps } n_{s} \\
& A(5, n)= \text { rf phase, at upstream end, } \phi[\text { deg] }
\end{aligned}
$$

An $r f$ quadrupole is a device that has a time-varying quadrupole electric field. The amplitude of the focusing field is unit?ess and is given by

$$
B=\frac{q \lambda^{2}}{m c^{2}} \frac{v}{r_{0}^{2}},
$$

where $V$ is the maxinum intervane voltage, and $r_{0}$ is the half-aperture of the vanes. 
TRACE can accommodate three forms for B: a constant, a linear taper, and an S-shaped curve. If the amplitude of the focusing field is constant $\left(B_{1}=B_{2}\right)$, there is no taper and the element is simply an rf quadrupole. If $B_{1} \neq B_{2}, B$ is assumed to have a linear taper from $B_{1}$ to $B_{2}$ over the length of the element, if the number of steps $\left(n_{s}\right)$ is entered as a positive number. When $n_{5}$ is entered as a negative number, an $S$-shaped focusing field model is used between $B_{1}$ and $B_{2}$, permitting the simulation of special radial matching sections. The field shape of this nonlinear model for an entrance radial matching section (denoted by $B_{1}=0, B_{2}>0$ ) is expressed as

$$
B=0.75 B_{2}\left[\cos \frac{\pi}{2}\left(\frac{z}{L}-1\right)+\frac{1}{3} \cos \frac{3 \pi}{2}\left(\frac{z}{L}-1\right)\right] \text {, }
$$

and for an exit section (denoted by $B_{1}>0, B_{2}=0$ )

$$
B=0.758,\left[\cos \frac{\pi}{2}\left(\frac{z}{L}\right)+\frac{1}{3} \cos \frac{3 \pi}{2}\left(\frac{z}{[}\right)\right],
$$

where $L=n_{B \lambda} B \lambda$, the length of the element.

The time-varying force constants depend on both $z$ and $t$, and are given by

$$
\begin{aligned}
& k_{e, x}{ }^{2}(z, t)=\frac{B(z)}{(B \lambda)^{2} \gamma} \cos (\omega t+\phi), \quad \text { and } \\
& k_{e, y}{ }^{2}(z, t)=\frac{-B(z)}{(B \lambda)^{2} \gamma} \cos (\omega t+\phi),
\end{aligned}
$$

where $w$ is the angular frequency of the $r f$ field, $t$ is the time and assumed to be zero when the beam is at the upstream end of the element (at $z=0$ ), and $\phi$ is the phase of the $r f$ when $t=0$. By assuming that the particles are synchronous with the $r f$, wt can be replaced by $2 \pi z / B \lambda$, and the external force can be considered to be a function only of $z$, the longitudinal position of the beam within this element.

TRACE uses average values for $k_{e, x^{2}}$ and $k_{e, y^{2}}$ for each computational interval. Average values are calculated by integrating the above expressions over each interval $\Delta z$, where $L$ is the length of the element:

$$
\Delta z=\frac{L}{\ln _{s} \top} \text {. }
$$


Let $z_{1}$ and $z_{2}$ denote the beginning and end locations of one interval, and let $\theta_{i}$ and $b_{i}$ be defined as follows:

$$
\theta_{\mathbf{i}}=2 \pi z_{\mathbf{i}} / R \lambda+\phi,
$$

and

$$
b_{i}=\frac{B\left(z_{j}\right)}{(B \lambda)^{2} \gamma} \quad \text { for } i=1,2 .
$$

Then, the $x$-component of the average external-field force over the interval $z_{1}$ to $z_{2}$ is

$$
\begin{aligned}
\overline{k_{e, x}{ }^{2}} & =\frac{1}{\Delta \theta} \int_{\theta_{1}}^{\theta_{2}^{2}}\left[b_{1}+\frac{\left(b_{2}-b_{1}\right)}{\Delta \theta}\left(\theta-\theta_{1}\right)\right] \cos \theta d \theta \\
& =\frac{1}{\Delta \theta}\left[b_{2} \sin \theta_{2}-b_{1} \sin \theta_{1}+\left(b_{2}-b_{1}\right)\left(\cos \theta_{2}-\cos \theta_{1}\right) / \Delta \theta\right],
\end{aligned}
$$

where $\Delta \theta=\theta_{2}-\theta_{1}$.

The $y$-component is

$$
\overline{k_{e, y^{2}}}=\overline{-k_{e, x^{2}}} \text {. }
$$

9. The rf Gap

$$
\begin{aligned}
& A(1, n)=\text { acceleration gradient } E_{0} T[M V / m] \\
& A(2, n)=\text { energy gain per cell } \Delta W[\mathrm{MeV}] \\
& A(3, n)=\text { synchronous phase angle } \phi_{S}[\mathrm{deg}]
\end{aligned}
$$

This element will model an acceleration gap in a drift-tube linac (OTL). There are three input parameters required to specify the rf gap; in addition, a value for the $r f$ wavelength is required (a general input parameter).

The accelerating gradient $E_{0} T$ is the product of the average ay ial electric field $E_{0}$, and the transient time factor $T$; $\Delta W$ is the energy gain in MeV for synchronous particles; and $\phi_{S}$ is the synchronous phase. The $r f$ gap is 
assumed to be a thin lens and is treated by setting up the transfer matrices as follows:

$$
R_{x}=R_{y}=\left[\begin{array}{cc}
1 & 0 \\
\frac{\Delta}{(B \gamma)_{\text {out }}} & \frac{(B y)_{\text {in }}}{(B y)_{\text {out }}}
\end{array}\right] \text {. }
$$

where, using average values for $\beta$ and $\gamma$,

$$
\Delta=-\frac{\pi q E_{0} T \sin \phi_{s}}{m_{0} c^{2} \bar{\beta} \bar{\gamma}^{2}} .
$$

10. Thin Leris

$$
A(1, n)=\text { reciprocal for the focal length } 1 / f[1 / m]
$$

The only parameter required for the thin lens is the focusing strength, the reciprocal of the focal length. For positive beams, a positive value means focusing in the horizontal plane; a negative value results in defocusing in the horizontal plane.

The transfer matrices are

$$
\begin{aligned}
& R_{x}=\left[\begin{array}{cc}
1 & 0 \\
-1 / f & 1
\end{array}\right], \text { and } \\
& R_{y}=\left[\begin{array}{cc}
1 & 0 \\
1 / f & 1
\end{array}\right] .
\end{aligned}
$$

11. Accelerator Tank

$$
\begin{aligned}
& A(1, n)=\text { length } \ell_{t}[\mathrm{~mm}] \\
& A(2, n)=\text { synchronous phase angle } \phi_{s}[\mathrm{deg}] \\
& A(3, n)=\text { number of cells } n_{C} \\
& A(4, n)=\text { input energy } w_{i}[\mathrm{MeV}] \\
& A(5, n)=\text { output energy } w_{0}[\mathrm{MeV}]
\end{aligned}
$$


This element simulates the effect of a coupled-cavity linac tank on the transverse beam dynamics. A coupled-cavity tank consists of a sequence of identical cells having a phase shift of $\pi$ between adjacent accelerating cells. Examples of such structures are side-coupled linacs and disk-and-washer linacs.

The required parameters are $\ell_{t}$, the tank length; $\phi_{s}$, the synchronous phase; $n_{c}$, the number of cells; and $W_{i}$ and $W_{0}$, t'e input and output kinetic energies in million electron volts.

Instead of having equations of motion in the form $x^{\prime \prime}+k_{x}{ }^{2} x=0$, we have $\frac{d}{d z}\left(B \gamma x^{\prime}\right)+B \gamma x_{x}^{2} x=0$,

which takes into account the change in $B_{\gamma}$ as the beam goes through the tank. Then we have equations like

$$
x^{\prime \prime}+2 a x^{\prime}+k_{x}^{2} x=0
$$

where

$$
2 a=\frac{1}{B r} \frac{d}{d z}(B \gamma)
$$

Assuming that "a" is a constant, the solution to this differential equation is

$$
x=c_{1} e^{r_{1} z}+c_{2} e^{r_{2} z}
$$

where

$$
r_{1}=-a+\sqrt{a^{2}-k_{x}^{2}}, \quad \text { and } \quad r_{2}=-a-\sqrt{a^{2}-k_{x}^{2}} \text {. }
$$

The initial conditions $x=x_{0}, x^{\prime}=x_{0}^{1}$ at $z=0$ allows us to evaluate $c_{1}$ and $c_{2}:$

$$
c_{1}=\frac{x_{0}^{\prime}+x_{0}\left(a+k_{x}^{\prime}\right)}{2 k_{x}^{\prime}}, \quad \text { and } \quad c_{2}=\frac{-x_{0}^{\prime}+x_{0}\left(-a+k_{x}^{\prime}\right)}{2 k_{x}^{\prime}} \text {, }
$$

where

$$
k_{x}^{\prime}=\sqrt{a^{2}-k_{x}^{2}}
$$


which is the square root of the modified force constant. The modified force constant includes the effects of the external field, space-charge field, and energy gain. The transformation matrix for a step $\Delta z$ is

$$
R=e^{-a \Delta z}\left[\begin{array}{ll}
R_{11} & R_{12} \\
R_{21} & R_{22}
\end{array}\right] \text {, }
$$

where

$$
\begin{aligned}
& R_{11}=\cosh \left(k_{x}^{\prime} \Delta z\right)+\frac{a}{k_{x}^{\prime}} \sinh \left(k_{x}^{\prime} \Delta z\right), \\
& R_{12}=\frac{1}{k_{x}^{\prime}} \sinh \left(k_{x}^{\prime} \Delta z\right), \\
& R_{21}=k_{x}^{\prime} \sinh \left(k_{x}^{\prime} \Delta z\right)\left[1-\left(a / k_{x}^{\prime}\right)^{2}\right], \quad \text { and } \\
& R_{22}=\cosh \left(k_{x}^{\prime} \Delta z\right)-\frac{a}{k_{x}^{\prime}} \sinh \left(k_{x}^{\prime} \Delta z\right) .
\end{aligned}
$$

The coefficient $e^{-a \Delta z}$ is a multiplier of each element in the matrix and is approximated by

$$
\mathrm{e}^{-\alpha \Delta z} \approx\left[\frac{(\beta \gamma)_{\text {in }}}{(B \gamma)_{\text {out }}}\right]^{1 / 2},
$$

the square root of the ratio of the value of $B_{\gamma}$ at the beginning and end of the step.

The force constants for the external field are

$$
k_{e, x}{ }^{2}=k_{e, y}{ }^{2}=\frac{\pi E_{0} T \sin \phi_{s}}{m_{0} c^{2} \lambda(B \gamma)^{3}},
$$

where

$$
E_{0} T=\frac{W_{0}-W_{i}}{\ell_{t} \cos \phi_{s}}
$$


Because $\phi_{s}$ is negative, $k_{e, x^{2}}$ is negative.

12. Accelerator Column

$A(1, n)=$ aperture radius of column $r_{a}[\mathrm{~mm}]$

$A(2, n)=$ length of column $\&[\mathrm{~mm}]$

$A(3, n)=$ change in energy $\Delta W[\mathrm{MeV}]$

$A(4, n)=$ number of steps $n_{S}$

$A(5, n)=$ current $I[\mathrm{~mA}]$

The accelerator column in TRACE consists of two thin lenses (TL), an entrance lens and an exit lens, separated by a distance (the length of the column) in which the energy change is linearly increased (or decreased) to the final value. The same cubic function used in the code SPEAM ${ }^{4}$ is used to smooth the potential function $\phi$ in calculating the fringe field:

$$
\phi=w_{0}+{\frac{z}{3 r_{a}}}^{3} r_{a} \Delta w / l \text {. }
$$

This function is integrated a distance twice the aperture radius before the column, and one aperture radius into the column as shown in Fig. 2. The reverse is true as you exit the column. The function is converted tc a focal length for a thin-lens approximation. For further information on this formulation, see Appendix B.

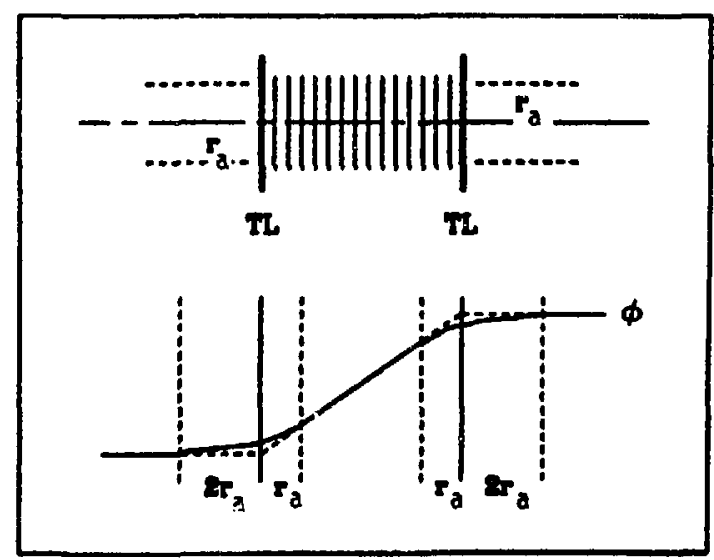

Fig. 2.

TRACE column model with cubic smoothing function. 
It has been found that extending the space charge from one to two aperture radii outside of the column more closely models experimental results. This space-charge extension can be done by entering current-change elements both before and after the column with one to two aperture-radii drifts between the column and the current-change cards. This extension can be noted in the graphic displays included in Appendix E, "Sample Output" (Figs. E-1 to E-3).

\section{Current Change}

$A(1, n)=$ amount of current to add or subtract $\Delta I[\mathrm{~mA}]$

The effective current (or space charge) can be modified at any point along the beamline with the current-change element. The amount of current in milliamperes to be added or subtracted from the beam current is specified in the first and only parameter of this element. The current is cumulative along the transport, starting with the initial effective beam current, XI (a general input parameter). Note that current-change cards are ignored if they are downstream of the buncher/debuncher simulator element.

$$
\begin{aligned}
& \text { 14. Horizontal R-Matrix } \\
& \hline A(1, n)=R_{11} \\
& A(2, n)=R_{12} \\
& A(3, n)=R_{21} \\
& A(4, n)=R_{22}
\end{aligned}
$$

It is possible to directly enter the beam-transformation matrix ( $R$-matrix) to represent any kind of transport element or beam transformation. The parameters are the inputs for the horizontal-plane R-matrix: $R_{11}, R_{12}$, $R_{27}$, and $R_{22}$. The next element, Element 15 , has the inputs for the vertical plane. The input values are based on units of meters and radians (a departure from the normal input units in TRACE). Element Types 14 and 15, immediately following each other, essentially form a single transformation matrix. Note that the graphic representation of an arbitrary R-matrix has no length; therefore, the beam envelope may reflect a discontinuity at the point of the R-matrix element (see Fiq. E-2). 


$$
\begin{aligned}
& A(1, n)=R_{33} \\
& A(2, n)=R_{34} \\
& A(3, n)=R_{43} \\
& A(4, n)=R_{44}
\end{aligned}
$$

See explanation above for horizontal $R$-matrix.

16. Permanent-Magnet Ouadrupole

$$
\begin{aligned}
& A(1, n)=\text { field gradient } B^{\prime}[T / m] \\
& A(2, n)=\text { physica] length } \ell_{p}[\mathrm{~mm}] \\
& A(3, n)=\text { inner radius } r_{i}[\mathrm{~mm}] \\
& A(4, n)=\text { outer radius } r_{0}[\mathrm{~mm}] \\
& A(5, n)=\text { distance to field terminator } s_{t}[\mathrm{~mm}] \text { (optional) }
\end{aligned}
$$

The field of a rare-earth cobalt (REC) PMQ of standard design is known analytically and has been verified experimentally.5 A discussion of the field formula is given in Appendix $C$.

Typically, PMQs are quite short and have strong pole-tip fields, resulting in "soft-edge" fringe fields that effectively extend out a distance two to three times the inner quadrupole radius (see Fig. 3 ).

The program defaults on a field extension factor of two and one-half times the inner radius; however, this factor, PQEXT, can be varied (see General Input, Sec. IV). The POEXT factor controls the extent or distance through which the program calculates the strength of the fringe field. Depending on the PQEXT, the program determines the length through which the fringe field will be calculated (shown qraphically by dashed lines surrounding the element) and superimposes the fringe field on adjacent elements including drifts, quadrupoles, bending magnets, columns, solenoids, and wigglers. * The superimposing is accomplished by adding the "k $\mathrm{e}^{2 "}$ force factors of the fringe field to that of any adjacent element(s) inside the PQEXT range when calculating the beamtransformation matrix. If there is more than one PMQ and they are within each other's fringe fields, the quadrupoles are grouped and their fields added before superimposing the field on other elements. Groupings are shown graphically when only one field center line exists for several PMQs between the field extension lines (see Fig. E-3).

\footnotetext{
FThe CDC 7600 version only.
} 


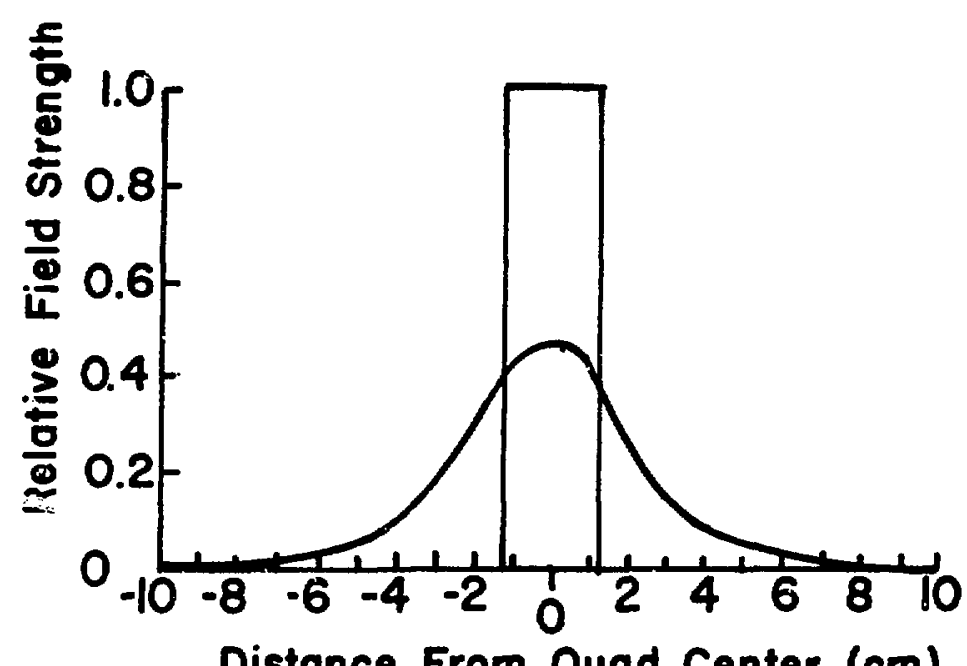

\section{Distance From Quad Center $(\mathrm{cm})$}

Fig. 3 .

Typical PMQ fringe field with a $2-\mathrm{cm}$ inner radius.

The first input parameter, the field gradient $[\mathrm{T} / \mathrm{m}]$, is the same as that for a quadrupole. The second parameter, the physical length of the quadrupole [mm], is also the effective length. The third and fourth parameters, the inner and outer radii of the quadrupole $[\mathrm{mm}]$, provide the information necessary to calculate the effective length and strength of the fringe field within the desired extension. The fifth parameter, the field terminator, allows the option of truncating the fringe field on one side of a PMQ (or group of PMQs) to allow modeling metal plates or other nearby materials that might terminate magnetic fields. The default is zero or no truncation. Note that PMQ fields extending outside the beamline are truncated and not included in calculations.

\section{GENERAL INPUT}

A. Beam Parameters

BEAMI (4) - Characterizes the initial or input beam at the starting elerient (N1) using the Twiss parameters (Courant-Snyder notation) as follows:

$$
\begin{aligned}
& \operatorname{BEAMI}(1)=\alpha_{x} \\
& \operatorname{BEAMI}(2)=\beta_{x}[\mathrm{~mm} / \mathrm{mrad}] \\
& \operatorname{BEAMI}(3)=\alpha_{y} \\
& \operatorname{BEAMI}(4)=\beta_{y}[\mathrm{~mm} / \mathrm{mrad}]
\end{aligned}
$$


EXI - Initial emittance in the horizontal plane at the starting element (N1) in units of millimeter-milliradians [mm-mrad]. Note that EXI should be four times the rms value when spacecharge forces are involved.

EYI - Initial emittance in the vertical plane at the starting element (NI) in units of millimeter-milliradians [mm-mrad]. Note that EYI snould be four times the rms value when spacecharge forces are involved.

W - Kinetic energy of the beam at the starting element [MeV].

ER - Rest energy of the beam particles [MeV].

Q - Charge state of beam particles (+1 for protons, -1 for electrons, etc.)

$X I$ - Effective beam current or space charge [mA] at the starting element.

DPOP - Momentum displacement $(\Delta \mathrm{p} / \mathrm{p})$ of the beam in per cent $[\%]$.

B. Fitting Parameters

BEAMF(4) - Final Beam - Characterizes the desired beam or beam parameters to be fitted at the final element (N2), using either the Twiss parameters or the beam-matrix notation depending on the type of matching involved. See Appendix F, Fig. F-3, or Sec. VI.A of this report for the BEAMF values corresponding to each type of matching.

MT - Matching Type - Flags the type of matching or fitting to be performed. There are eight types of matching available (numbered 1 to 8 ) that are explained in detail in Sec. VI.A of this report and are summarized in Appendix F, Fig. F-3. 
ME(4) - Matching Element - Element number of those elements designated as variables for matching. For matching Types 1,4 , and 6 , four MEs (variables) are required; for the remaining matching types, only two MEs are required.

MP(4) - Matching Parameter - The parameter number ( 1 to 5 ) that designates the parameter value of the corresponding ME to se varied. If no parameter number is specified, the program defaults on 1 , meaning that the first parameter of the ME will be varied. As with the ME input, the number of MPs required (two or four) depends on the type of matching and is the same as that for the MEs.

MC - Matching Constraint - Allows the pairing of two of the variables used for matching so that incremental changes made in the values of the two matching variables are either equal or opposite. The MC is invoked by entering a two-digit number that represents the two MEs $[\operatorname{ME}(1),(2),(3)$, or (4)] to be linked togetrer. Any two-digit combination of the numbers 1 to 4 is aciepted. A negative entry will link the two matching variables inversely, which will allow fitting with drifts, but. will maintain constant beamline length. Once set, the MC remains in effect until turned off by entering a zero. Note that $M C$ values are not stored when files are saved. Also, the MC entry is ignored with matching Types 5 or 6 .

CMV(4) - Couple Matching Variables - Allows the coupling of any or all of the MEs to any transport-element parameter in the beamline.

A typical entry may be as follows:

$$
\operatorname{CMV}(2)=A(1,16)
$$

Any entry between $A(1,1)$ and $A(5,50)$ or $-A(1,1)$ and $-A(1,50)$ is accepted. In the above example, Matching Variable 2 is coupled to the first parameter of Element 16; concequently, both will vary with the same magnitude and direction. A negative entry couples inversely, resulting in equal changes in magnitude but in opposite directions. Inverse coupling 
enables fitting with drifts, maintaining constant overall beamline length. CMV values are stored when files are saved. To cancel a CMV input, enter nothing or a blank. Coupling entries are ignored with matching Types 5 or 6.

NIT - Number of Iterations - NIT sets the maximum number of iterations in the matching process, after which the best solution is chosen and the matching process terminated. The default value of NIT is 10 iterations. For NIT $\geq 50$, or if space charge is involved, a bell rings at the termination of the matching process because the time involved in the calculations can extend beyond 1-2 min, depending on the type of computer. The user should note that small values of NIT (10 $\leq$ NIT $\leq 20)$, with frequent initiations of the matching process, are usually more productive than large values of NIT because the initiation of the matching process calls for new sets of random numbers and helps avoid stagnation in local minima during calculations.

CC - Convergence Criterion - Refers to the maximum mismatch factor (defined in Sec. VI.B) acceptable in the solutions generated to terminate matching during the matching process. The default value is 0.01 for all types of matching except Type 6 , where the default is 0.0001 . When a solution is generated during the matching process whose mismatch factor is less than or equal to the maximum acceptable mismatch factor, the matching process is terminated and a double-bell rings at the termina't.

CONV(4) - Conversion Factor - Used only for the display of the "Values" under "Matching Variables" on the graphic output. Allows the user to print out the matching variable values in units other than those used by TRACE. The value identified by ME(n) and $\operatorname{MP}(n)$ is multiplied by $\operatorname{CONV}(n)$ when displayed on the graphic output. 
$X M$ - $X_{\max }$ - Sets the scale for $X_{\max }$ and $Y_{\max }$ on the phase-space plots in millimeters $[\mathrm{mm}]$. This value is printed below the right-hand phase diagrams on the graphic output (see Figs. E-1 to E-3).

$X P M$ - $X_{\text {max }}^{\prime}$ - Sets the scale for $X_{\text {max }}^{\prime}$ and $Y^{\prime}{ }_{\max }$ on the phase-space plots in milliradians [mrad]. This value is printed below the right-hand phase diagrams on the graphic output (see Figs. $E-1$ to $E-3$ ).

XYM - Beam size maximum - Sets the scale for the maximum beam radius that can be shown on the profile graph in millimeters [mm]. This value is printed in the upper left-hand corner of the profile box on the graphic output (see Figs. E-1 to E-3).

PE(6) - Printout Element - Element number of those elements selected to have their values printed on the graphic output under "Printout Values". Provides information on grapnic output for user convenience (see Figs. E-1 to E-3).

PP(6) - Printout Parameter - The parameter number ( 1 to 5 ) that designates the parameter value of the corresponding printout element to be listed on the graphic output under "Printout Values." If no number is specified, the program defaults on 1.

PCONV(6) - Conversion Factor - Used only for the display of "Values" under "Printout values" on the graphic output. Allows the user to display parameter values in units other than those used by TRACE. The value identified by $P E(n)$ and $P P(n)$ is multiplied by $\operatorname{PCONV}(n)$ when displayed on the graphic output.

There are margins setting the height and width of the graphic display that are not input values, but that can be changed by altering the data file with an editor. The values are in 
Lines 12 and 13 of the ASCII data file shown in Fig. E-6. MRG(1-4) are, respectively, the left-hand, right-hand, lower and upper limits of the graphic display in units of the Tektronics 4000 Series terminal. IXW and IYW control the dimensions of the phase-space diagrams and IYP the height of the profile box.

D. Other

N1 - Number of the starting elemest where the transport calculations and beam trace begin. At $N 1$, all initial beam conditions are applied (for example, BEAMI, EXI, EYI, XI, W, etc.) For forward beam calculations, N2 is greater than or equal to N1. If $N 1=N 2$, calculations are in the forward direction through one element only.

N2 - Number of the final element through which transport calculations and beam trace terminate. At $N 2$, the output beam, BEAMO is printed out. If $N 2 \geq N 1$, beam trace is forward; if $N 2<N 1$, beam trace is backward. $N 1$ is automatically adjusted if $N 2$ is changed to reverse beam trace. To avoid this automatic adjustment, enter both $\mathrm{N} 1$ and $\mathrm{N} 2$ together on the same input line.

SMAX - Maximum computational step size in millimeters. Any element in the beamline whose length is longer than SMAX is divided into an integer number of segments of equal length $\leq$ SMAX. At each step, the linear transformation matrix ( $R$-matrix) is calculated (including space-charge forces and superimposed PMQ fields, if any).

WAVEL - Wavelength in millimeters [mm] of the rf in the transport system. This value is only necessary when using elements that require $r f$. If other than zero, this value is printed in the lower left-hand corner of the profile box on the graphic output (see Fig. E-2). 
PQEXT - Permanent-Quadrupole Extension Factor - PMQ fringe fields extend out beyond the physical length of the quadrupole, equal to a factor (PQEXT) times the quadrupole inner radius. Unless a value is entered, the program defaults on a value of 2.5 . The extent of the fringe field for calculation purposes is shown graphically by dashed lines on either side of the quadrupole. The value of PQEXT is displayed with the data in the center of the graphic output (See Figs. E-1 to E-3).

CMT(50) - In-line comments for each element in the data file. Al lows the user eight characters per element to identify each element in the data file if desired. See Fig. E-4 for an example of in-line comments.

COMMENT - Data file comment for entire file. The first line of a TRACE data file (see Fig. E-6) defaults on the statement "Remember units are $\mathrm{mm}$ and $\mathrm{mrad}$ (quadrupole $=T / \mathrm{m}$ )." This can. be changed by making an eritry, up to 60 characters. This comment appears at the end of a printout from a "p" command (Fig. E-4), and can also be displayed on the graphic output with the "C" command (see Figs. E-1 to E-3).

\section{OPERATING INSTRUCTIONS}

A. Using the Code

TRACE was designed to be used on small computers and was intended to address small or limited beam-dynamics problems that require no more than simple first-order approximations. Therefore, running TRACE is different from most other beam-transport codes in that all work is done interactively. From a user's viewpoint, this is often highly desirable. Typing a single keyboard character can effect calculations, printouts, and/or graphic display. The versatility of the command procedure allows one to easily exchange data, run the beam from any one point to another, run forward or backward, do fitting calculations at any point, etc. The output may prompt the user to alter the beamline or to modify a parameter value and recalculate the output beam. There is no need to open and/or alter a data file; all data manipulation and calculations 
are performed on-line in an intaractive mode, giving immediate results. Data files can be read, altered, or rewritten as desired from the interactive mode. When a user begins to run TRACE, he is asked to supply the name of a data file. If there is no data file to start with, the program will provide some "dummy" data (a transport system with three drifts), allowing access to the program and the HELP package and the opportunity to build a transport file. Once into the program, the cursor cross hairs appear, indicating the "wait-for-command" mode. The program is then operated by entering a command (that is, typing a single character); when the cross hairs reappear, the program is ready to accept another command. "Type-ahead" terminals may enter a sequence of commands, and when all are completed, the cross hairs reappear.

A list of commands recognized by TRACE can be seen in Fig. 4, which is itself part of the on-line HELP package accessible from the interactive mode by typing "H." Output beam parameters (BEAMO) can be requested at any point along

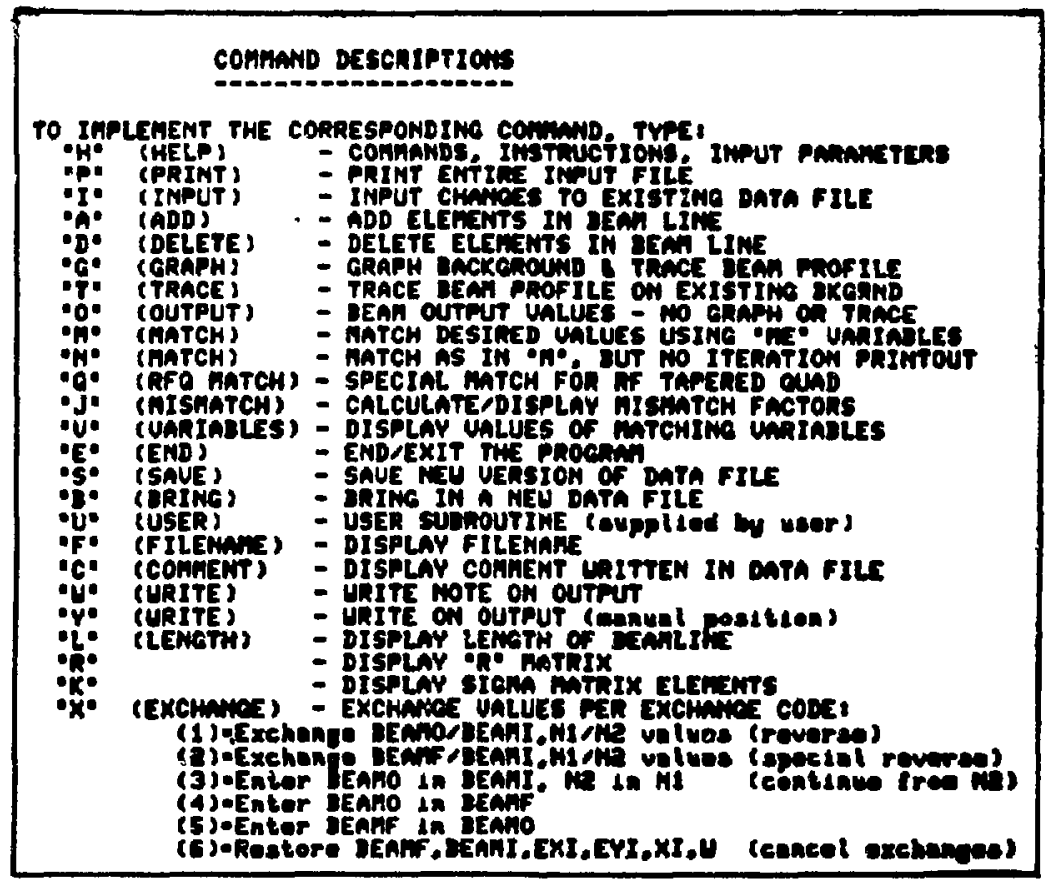

Fig. 4.

List of commands from the TRACE HELP package.

\footnotetext{
*Information is automatically printed down the left-hand side of the screen beginning at the location of the horizontal cross hair; therefore, placing the horizontal cross hair near the top of the screen will utilize maximum space. Moving the cross hairs resets the starting position.
} 
the transport system by typing "O"; in similar fashion with a different command, the user can obtain the beam matrix, the transformation matrix, the length of the beamline, the beam profiles or phase ellipses, and the mismatch factor. All information is printed beside the graphic display, leaving a complete record of all transactions, modifications, and resulting calculations down the left-hand side of the page (see Fig. E-1).

Pages 1, 2, and 3 of the TRACE HELP package are reproduced in Appendix $F$ as Figs. F-1, F-2, and F-3. To access the HELP package from the program, type an "H" from the cross-hair mode. The pages of the HELP package are displayed in succession. The first page lists the commands available to the user along with the general input parameters discussed earlier in Sec. IV. The second page lists all of the transport system elements and their parameters as discussed in Sec. III. The third page summarizes the fitting capabilities of the program as described in the following section (Sec. VI).

\section{B. Description of Interactive Commands}

\section{Data I/0}

"P" (PRINT) - Prints out most of the data in the data file. If the cross hairs are moved previous to this command, the printout begins at the cross-hair junction. If the cross hairs are not moved, the printout location defaults to the top, right-hand side of the page. See Fig. E-4 for a printout of the data file using this command.

"I" (INPUT) - Allows data to be entered or changed in the data file in a namelist-type manner (that is, variable=value; variable= value; or subscripted variable=value; value;value). Note that input fields must be separated by semicolons $(;)$. Up to 80 characters may be entered on one line with fields separated by semicolons. The input routine checks subscripts and variable names and informs the operator of any errors. The only inputs rejected are those for. which an error message has been generated. All others on the input 
line are accepted. All subscripted variables entered without a subscript are assumed to have a subscript of (1). A sample input line is shown in Figs. E-1 and E-4.

"A" (ADD) - Allows the user to add transport elements anywhere in the beamline. The program prompts the user to enter two numbers: $M I$ (the number of elements one wants to add), and M2 (the sequential number of the element in the beamline before which the additional elements are to be inserted). After the user supplies this information, the program renumbers all of the transport elements, enters the input mode, and asks for the element types of the new elements, starting with the first one. It remains in the input mode until the user has made at least as many entries as the number of e lements added.

"D" (DELETE) - Makes possible the deletion of any number of transport elements from any point in the beamline. The prompt is for two numbers: $M$ I (the number of elements to be deleted), and $M 2$ (the sequential number of the first element in the beamline to be deleted).

"S" (SAVE) - Writes a new data file with the current information, allowing the user to write it as a new version of the same file, or to enter a new filename.

"B" (BRING) - Brings in another data file, replacing all current information with data from the new file. The program prompts the user for the name of the file to be read.

\section{Calculations}

"G" (GRAPH) - Erases the screen, draws the graphic background for the phase diagrams and transport line, and calculates and draws both the beam envelope through the transport line and the phase-space ellipses at the beginning and end of 
the beamline. The information printed on the graphic output includes input and output data such as the beam parameters (BEAMI, BEAMO), effective current ( $I$ ), energy ( $W$ ), and emittance (EX, EY). If there is a matching type, the MEs and their values, along with the desired output beam values (BEAMF), are also printed out. In addition, if N2 is equal to the last element in the beamline, the mismatch factor(s) is included.

"T" (TRACE) - Traces (calculates and draws) the beam envelopes through the transport line as well as the phase-space ellipses at both the beginning and the end. This command is used after the "graph" command and is useful for comparing beam envelopes and phase ellipses when changes are made in element field strengths, input beam, current, emittance, etc. (see Fig. E-3). For graphic display purposes, this command assumes no change has been made in the number, length, or position of elements in the beamline.

"0" (OUTPUT) - Calculates the output beam (BEAMO) only; no graphics involved.

"M" (MATCH) - Initiates fitting calculations to find desired matched beam. Uses date and time for a seed number to generate random numbers for possible solutions (VAX version on ly). Prints out the mismatch factor (first column) and the matching variable values (remaining columns) for the initial solutions and each succeeding iteration. The printout location depends on the location of the cross hairs. The final row of numbers is the chosen solution.

"N" (MATCH) - Initiates fitting calculations identical to the above "M" command except for the printout. Only the initial and final mismatch factors are displayed, with no intermediate iterations shown. An example is seen in Fig. E-3. To see the current or new values of matching variables, use the "V" (variables) command. Print location is automatic. 
"J" (MISMATCH) - Calculates and displays the mismatch factor(s) between the desired beam (BEAMF) and the beam at N2 (BEAMO).

"Q"(RFQ MATCH) - Special matching routine for an RFQ entrance or exit only. Requires a matched beam to start with (MT $=6$ ) in a periodic system, which is then projected backward through an $r f$ tapered quadrupole from each section in the first $360^{\circ}$ (one $B \lambda$ length). The code averages the results of these backtraces to produce a beam at the entrance (or exit) of the RFQ. The mismatch factor calculated is the worst case of the averaged beam compared to the calculation at each phase. Figure E-5 shows the output generated from this command.

3. Other

" $X$ " (EXCHANGE) - Provides for a quick exchange of beam parameters $\alpha_{x}$, $\beta_{x}$, $\alpha_{y}, \beta_{y}$, and other important values. Prompts the user for one of the following exchange codes to be entered:

Exchange Code 1 reverses beam. Exchanges BEAMO and BEAMI, $N 1$ and N2, and the initial and final emittances, beam current and energy. Before reversing beam, calculations must be made in one diraction.

Exchange Code 2 reverses beam with matched parameters. Exchanges BEAMF and BEAMI, $N 1$ and $N 2$, and the initial and final emittances, beam current, and energy. Same as Exchange Code 1 above, except that it uses BEAMF instead of BEAMO. Useful for finding matched solutions in reverse.

Exchange code 3 continues beam from stopping point. Enters BEAMO in BEAMI, and $\mathrm{N} 2+i$ in $N 1$, where $i= \pm 1$ depending on the direction of travel. The output beam at $\mathrm{N} 2$ becomes the starting beam at that location, now N1. The user is prompted to enter a new N2 to indicate how far to 
go and in which direction. This command can be quite.useful in reducing the time required during matching calculations by shortening the beamline to only that portion where the matching variables are located.

Exchange Code 4 enters output beam (BEAMO) parameters ( $\alpha_{X}$, $\left.\beta_{x}, \alpha_{y}, \beta_{y}\right)$ into the desired matched beam (BEAMF).

Exchange Code 5 enters desired matched beam (BEAMF) parameters into the output beam (BEAMO).

Exchange Code 6 cancels exchanges. Restores all values (BEAMI, BEAMF, N1, N2, XI, W, EXI, EYI) to the original values read from the data file. These values remain unchanged until a new data file is written.

"R" (R-MATRIX) - Displays the transformation matrix between $N 1$ and N2 generated from the previous run (the last "T", "G", " 0 " or " $J$ " command). The units of $R_{12}$ and $R_{34}$ are in meters; $R_{21}$ and $R_{43}$ are in reciprocal meters; and the remaining matrix elements, $R_{11}, R_{22}, R_{33}$, and $R_{44}$, are unitless.

"K" ( $\sigma$-MATRIX) - Displays the diagonal elements of the "sigma" or beam matrix $\left(\sqrt{\sigma_{11}}, \sqrt{\sigma_{22}}, \sqrt{\sigma_{33}}, \sqrt{\sigma_{44}}\right)$ plus two of the correlation matrix elements $\left(r_{21}, r_{43}\right)$ at N2. These numbers describe BEAMO in the traditional TRANSPORT CODE notation (to obtain this notation for BEAMI, reverse the beam).

"V" (VARIABLES) - Displays the current values of the matching variables.

"F" (FILENAME) - Displays the name of the current data file.

"L" (LENGTH) - Calculates and displays the current beamline length. 
"C" (COMMENT) - Displays the comment line in the data file.

$$
\text { "E" (END) - Exits program. }
$$

"U" (USER) - Calls a user-supplied subroutine.

"W", "Y", "Z" - Writes notes on graphic output - "W" writes the word "Note:" and allows the user to type in a message; " $Z$ " is identical to "W" except that the word "Note:" is not printed; "Y" allows the user to print numbers or letters at the cross-hair position, which is useful for identifying multiple beam envelopes, phase ellipses, etc. (see Fig. E-3).

\section{vi. MATCHING CAPABILITIES}

\section{A. Types of Matching}

Eight types of matching criteria are allowed by TRACE arid are listed in Table II. Six of these types find proper values for a specified number of transport-system parameters (such as quadrupole strengths) that cause the beam to have the desired characteristics at a given location. The other two types find the proper input beam ellipse parameters at a certain location. All of these situations involve finding the solution to a set of either two or four nonlinear equations.

The method used is that of regula falsi, an iterative procedure which, starting with an initial "guess" for the solution, hopefully converges to a solution in a reasonable number of trials. A solution is assumed to have been found if a convergence criterion, a value calculated at each iteration, is less than the specified input value CC. If a solution has not been found after a given number of iterations (default value of 10 ), the procedure is terminated with the "best" solution that has been found. The procedure is easily restarted, the computer taking the best solution as the initial guess. The best solution is the one that most nearly meets the convergence criterion. 
TABLE II

TYPES OF MATCHING

\begin{tabular}{|c|c|c|}
\hline $\begin{array}{l}\text { ateh } \\
\text { jpe }\end{array}$ & $\begin{array}{c}\text { Deaired Values } \\
\text { to Mateh }\end{array}$ & Commeate \\
\hline 1 & $\alpha_{x} \beta_{x} \alpha_{y}, \beta_{y}$ & \multirow{4}{*}{$\begin{array}{l}\text { Fits } \\
\text { desired } \\
\text { values } \\
\text { at any } \\
\text { point in } \\
\text { beamline }\end{array}$} \\
\hline 2 & $\begin{array}{l}X^{\prime} \text { at } X \text { max } \\
Y^{\prime} \text { at } Y \text { max }\end{array}$ & \\
\hline 3 & $\begin{array}{l}X \text { max } \\
Y \text { max }\end{array}$ & \\
\hline 4 & $\begin{array}{l}\mathbf{X} \max \\
\mathbf{X} \\
\mathbf{X} \max \\
\mathbf{Y} \max \\
\mathbf{Y}^{\prime} \max \\
\end{array}$ & \\
\hline 5 & $\begin{array}{r}\alpha_{x} \quad \beta_{x} \\
(n 1=n 2)\end{array}$ & $\begin{array}{c}\text { Limiting } \\
\text { section: } \\
\text { beam clipping } \\
\left(X \text { and }\left(X^{\prime}\right)\right.\end{array}$ \\
\hline 6 & $\begin{array}{c}\text { Input and } \\
\text { output } \\
\text { beam } \\
\text { parameter }\end{array}$ & $\begin{array}{l}\text { For periodic } \\
\text { systom } \\
\text { (sete output } \\
\text { equal to input) }\end{array}$ \\
\hline 7 & $\alpha_{x}, \beta_{x}$ & \multirow{2}{*}{$\begin{array}{l}\text { For } \\
\text { solenoids }\end{array}$} \\
\hline 8 & $\alpha_{y}, \beta_{y}$ & \\
\hline
\end{tabular}

The eight types of matching criteria are as follows:

1. $(M T=1)$ The desired ellipse parameters $\left(\alpha_{x}, \beta_{x}, \alpha_{y}, \beta_{y}\right)$ are specified at the end of a transport element. Four parameters must be indicated as variables (ME/MP values). The calculated convergence criterion is the maximum of the mismatch factors in the $x-x^{\prime}$ and $y-y^{\prime}$ planes (the mismatch factor is defined later). 
2. (MT $=2$ 2) The desired values of $x^{\prime}$ at the horizontal edge of the bean $x_{\max }$, and of $y^{\prime}$ at $y_{\max }$ are specified. The calculated con. vergence criterion is the maximum of $\left|\Delta x^{\prime}\right|$ and $\left|\Delta y^{\prime}\right|$, wher $\Delta x^{\prime}$ and $\Delta y^{\prime}$ are the differences in the obtained values from the specified values if the specified values are $<1$ mrad. Otherwise $\Delta x^{\prime}$ and $\Delta y^{\prime}$ are relative errors. Two parameters must be specified as being variables (ME/MP values).

3. $(M T=3)$ The beam sizes, $x_{\max }$ and $y_{\max }$, are specified. The calculated convergence criterion is the maximum of $\left|\Delta x_{\max }\right|$ and $\left|\Delta y_{\max }\right|$, where these quantities are simple differences if the specified values are $<1 \mathrm{~mm}$, and relative errors otherwise. Two variables are required.

4. $(M T=4)$ The desired beam is specified by values of $x_{\max }, x^{\prime}{ }_{\max }$, $y_{\text {max }}$, and $y^{\prime}{ }_{\max }$ at the end of a transport element. Four variables are required. The calculated convergence criterion is

$F C=\left[\left(\frac{\Delta x_{\text {max }}}{x_{\text {max }}}\right)^{2}+\left(\frac{\Delta x_{\text {max }}^{\prime}}{x_{\text {max }}^{\prime}}\right)^{2}+\left(\frac{\Delta y_{\text {max }}}{y_{\text {max }}}\right)^{2}+\left(\frac{\Delta y_{\text {max }}^{\prime}}{y_{\text {max }}^{\prime}}\right)^{2}\right]^{1 / 2}$

5. (MT = 5) The object of matching Type 5 is to find the ellipse parameters $\alpha$ and $B$ (both $p l a n e s$ are assumed to be identical) at the entrance of a set of "limiting apertures" so that the beam can be scraped in an optimum fashion. ${ }^{6}$ In this case, the unknowns are $\alpha$ and $\beta$. The conditions to be fulfilled are

$$
\begin{aligned}
& f_{1} \equiv \alpha-R_{11}=0, \\
& f_{2} \equiv \beta-R_{12}=0,
\end{aligned}
$$

and

$$
t \equiv \frac{R_{11}-R_{12} \alpha / \beta}{R_{21}-R_{22} \alpha / \beta}=0,
$$


where $R_{i j}$ is the $i j$ element of the transfer matrix between the two apertures. The calculated convergence criterion is

$$
F C=f_{1}^{2}+f_{2}^{2}+t^{2}{ }^{1 / 2} .
$$

6. (MT = 6) The purpose of matching Type 6 is to find the matched ellipse parameters into a periodic system. The unknowns are $\alpha_{x}, \beta_{x}, \alpha_{y}$, and $\beta_{y}$, which are the values of both the input and output beam parameters for a section of a tran:port system. Nonzero starting values for $\alpha_{x}, \beta_{x}, \alpha_{y}$, and $B_{y}$ must be entered to start the calculation. The calculated convergence criterion is the maximum of the mismatch factors in both transverse planes. The mismatches are calculated between the input and output ellipses.

7. (MT $=7)$ Intended for solenoid transport systems, the desired values of $\alpha_{x}$ and $\beta_{x}$ are specified. Two parameters must be specified as variables. The calculated convergence criterion is the mismatch factor in the $x-x^{\prime} p l a n e$.

8. (MT $=8 ;$ Intended for solenoid transport systems, the desired values of $\alpha_{y}$ and $\beta_{y}$ are specified. Two variables are required, and the calculated convergence criterion is the mismatch factor in the $y-y^{\prime}$ plane.

Page 3 of the TRACE HELP nackage summarizes the above discussion of matching capabilities, indicating how to specify the desired beam parameters (using BEAMF) as well as how to specify the variables (using ME, MP). In addition, it is possible to introduce constraints on the variables through the use of the MC or match-constraint option or to couple the variables to any of the parameters in the beamline through the use of the CMV or "couple-matchingvariables" input. The match-constraint option links two of the matching variables, so that an incremental change in one equals the chanqe in the other. This is done by entering a two-digit number representing the two variables to be linked together. The couple-matching-variable option allows matching variables to be coupled to other parameters in the beamline through the use of the CMV input, providing greater flexibility. See "General Input," Sec. IV for a more detailed discussion. 


\section{B. Definition of Mismatch Factor}

As the name implies, the mismatch factor is a quantitative measure of the difference between two ellipses having the same area and the same center. If the two ellipses are not identical, one ellipse could be enlarged so that it just encloses the other ellipse; the amount of enlargement is related to the mismatch factor.

One way of looking at the problem is as follows: First, find the transformation that maps one of the ellipses into a circle, and apply the same transformation to the second ellipse to get a modified ellipse. Denote the radius of the circle by $R_{c}$ and the length of the major semiaxis of the ellipse by $R_{e}$ (see Fig. 5) . Because the ellipse and the circle have the same area, $R_{e}$ will be greater than or equal to $R_{C}$. The mismatch factor used in TRACE is

$$
M=\frac{R_{e}}{R_{c}}-1 \text {. }
$$

\section{Two ellipses that have the same area} transform to coordinate system in which one ellipse is a circle:
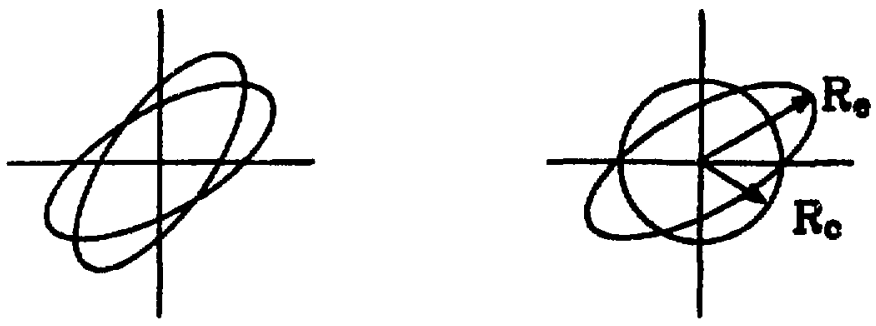

$$
M=\frac{R_{0}}{R_{0}}-1
$$

Fig. 5.

Definition of mismatch factor. 
Periodic beam-transport systems have matched conditions if they are stable. That is, if one matches a beam to the transport system, and if one could measure the size of the beam at the same location in each period of the system, then one would see a constant beam size. A mismatched beam would oscillate about this matched size, and at some places the beam would be larger by a factor $1+M$. For example, a mismatch factor of 0.1 , defined as above, would mean that the beam would occasionally be $10 \%$ larger than it would be if it were matched.

A word of caution is in order. It is quite common in the literature for the mismatch factor to be defined as $\left(R_{e} / R_{c}\right)^{2}-1$, which relates to how much the area of the circle would have to be increased to enclose the ellipse. If this definition is used, one would calculate a mismatch factor of 0.21 for the example given above. 


\section{APPENDIX A}

\section{EMITTANCE CALCULATIONS THROUGH THE BUHCHER}

Following Weiss, ${ }^{7}$ the increase in the transverse emittance of a cylin drical beam as it passes through a double-drift harmonic buncher is calcula as follows: Considering that all the bunching action takes place at the ha monic buncher gap (thin lens), we can express the divergence of a particle leaving the gap, $x_{f}^{\prime}$, in terms of the buncher parameters and the particle's displacement and divergence at the entrance to the gap

$$
x_{f}^{\prime}=x^{\prime}+x\left(k_{1} \cos \phi-k_{2} \cos 2 \phi\right) \text {, }
$$

where $\phi$ is the phase of the particle in the gap with respect to the fundame bunching frequency, and $k_{i}$ the transverse force constant of each of the bunchers

$$
k_{i}=\frac{\pi \Delta W_{i} T_{i}}{2 \beta \lambda W} \quad, \quad i=1,2 .
$$

In the above expression, $\Delta W_{1}$ and $\Delta W_{2}$ are the maximum energy gains at the fu mental and harmonic frequencies, respectively. The output displacement of particle is the same as its input displacement

$$
x_{f}=x
$$

The new emittance is found by calculating the new second momenta $x_{f}{ }^{2}$, $x_{f}^{\prime}$, , and $\left(x x^{\prime}\right)_{f}$. If the buncining efficiency is $n$, then one is usually interested only in the emittance of that portion of the beam having a phase spread at the buncher in the ranqe $-n \pi \leq \phi \leq n \pi$. Therefore, the averages are taken over this interval, with the average defined as

$$
\overline{f(n)} \equiv \frac{1}{2 n \pi} \int_{-n \pi}^{n \pi} f(\phi) d \phi .
$$


The second momenta of the output beam may be expressed as follows:

$$
\begin{aligned}
& \overline{x_{f}^{2}}=\overline{x^{2}}, \\
& \overline{x_{f}^{\prime 2}}=\overline{x^{\prime 2}}+\overline{2 x x}\left(k_{1} \cos \phi-k_{2} \cos 2 \phi\right)+\overline{x^{2}\left(k_{1} \cos \phi-k_{2} \cos 2 \phi\right)^{2}},
\end{aligned}
$$

and

$$
\overline{\left(x x^{\prime}\right)_{f}}=\overline{x x^{\prime}}+\overline{x^{2}\left(k_{1} \cos \phi-k_{2} \cos 2 \phi\right)} \text {. }
$$

Because the input co-ordinates $x$ and $x^{\prime}$ are assumed to be independent of $\phi$, the previous two equations may be written as

$$
\overline{x_{f}^{\prime 2}}=\overline{x^{\prime 2}}+2 A \overline{x x^{\prime}}+B \overline{x^{2}}
$$

and

$$
\overline{\left(x x^{\prime}\right)_{f}}=\overline{x x^{\prime}}+A \overline{x^{2}}
$$

By straightforward integration, one finds the coefficients to be

$$
A=k_{1} \frac{\sin n \pi}{n \pi}-k_{2} \frac{\sin 2 n \pi}{2 n \pi},
$$

and

$$
B=\frac{k_{1}{ }^{2}}{2}\left(1+\frac{\sin 2 n \pi}{2 n \pi}\right)+\frac{k_{2}{ }^{2}}{2}\left(1+\frac{\sin 4 n \pi}{4 n \pi}\right)-2 k_{1} k_{2}\left(\frac{\sin n \pi}{n \pi}-\frac{2}{3} \frac{\sin ^{3} n \pi}{n \pi}\right) .
$$

The transverse rms emittance after the buncher gap is

$$
\left(E_{f}\right)_{r m s}=\left[\overline{x_{f}^{2}} \overline{x_{f}^{\prime 2}}-\overline{\left(x x^{\prime}\right)_{f}^{2}}\right]^{1 / 2} .
$$


In TRACE, one is allowed to follow the beam upstream as well as downstream. If the beam passes through a butcher while going upstream, then the emittance is changed in the inverse manner. That is,

$$
\begin{aligned}
& \overline{x^{2}}=\overline{x_{f}^{2}}, \\
& \overline{\left(x x^{2}\right)}=\overline{\left(x x^{\top}\right)_{f}} \cdot A \overline{x_{f}^{2}},
\end{aligned}
$$

and

$$
\overline{x^{2}}=\overline{x_{f}^{2}}-2 A \overline{\left(x x^{1}\right)_{f}}+\left(2 A^{2}-B\right) \overline{x_{f}^{2}}
$$

41 


\section{APPENDIX B}

\section{ACCELERATOR COLUMN MODEL}

The accelerator column is modeled in TRACE by two electrostatic aperture lenses between which there is a constant accelerating field of $\Delta \mathrm{W} / \mathrm{ql}$ ( $\Delta \mathrm{W}$ = energy change, $\ell=$ column length, $q=$ charge state) and outside of which there is usually no electric field. The aperture lenses are treated as thin lenses and give the beam a focusing radial impulse at the entrance to the column and a defocusing radial impulse at the column exit. The length of the column is divided into $n_{s}$ equal-length segments. At each segment, the beam is drifted (with space charge) to the center of the segment, where the kinetic energy is changed by $\Delta W / n_{s}$, and then is drifted to the end of the segment.

In the paraxial approximation, the radial component of the electric field can be calculated from the on-axis longitudinal field by

$$
E_{r}(r, z) \approx-\frac{r}{2} \frac{d}{d z}\left[E_{z}(0, z)\right] .
$$

The radial component of the force is

$$
F_{r}(r, z)=a E_{r}(r, z)
$$

Except near the aperture, $E_{z}(0, z)$ is constant and therefore $E_{r}$ is zaro.

Concentrating on the entrance aperture, let $z_{1}$ denote the nearest longitudinal coordinate upstream of the aperture at which $E_{z}$ is zero (or at a constant value), and let $z_{2}$ denote the nearest downstream location where $E_{z}=\Delta W / q l$. The average radial force over this distance is

$$
F_{r}(r)=\frac{1}{\left(z_{2}-z_{1}\right)} \int_{z_{1}}^{z_{2}} F_{r}(r, z) d z=\frac{-q}{2\left(z_{2}-z_{1}\right)} \int_{z_{1}}^{z_{2}} r \frac{d}{d z}\left(E_{z}\right) d z .
$$

Assuming $r$ is constant over this interval,

$$
F_{r}=\frac{-q r}{2\left(z_{2}-z_{1}\right)}\left[E_{2}\left(0, z_{2}\right)-E_{z}\left(0, z_{1}\right)\right] \text {. }
$$


When $E_{z}\left(0, z_{1}\right)$ is zero, we have:*

$$
F_{r}=\frac{-r \Delta W / l}{2\left(z_{2}-z_{1}\right)} \text {. }
$$

The change in the $x$-component of the momentum because of this average radial field is

$$
\Delta p_{x}=F_{x} \Delta t=\frac{x}{r} F_{r} \int_{z_{1}}^{z} \frac{d z}{v} \approx-\frac{x}{2 \bar{v}} \Delta W / \ell,
$$

where $\bar{v}$ denotes the average velocity between $z_{1}$ and $z_{2}$. The change in $x^{\prime}$ is given by

$$
\Delta x^{\prime}=\frac{\Delta p_{x}}{p_{z}}=-\frac{x \Delta w / \ell}{2 \bar{v} m v_{0}},
$$

where $v_{0}$ is the initial velocity.

For very small apertures, $z_{1}$ and $z_{2}$ are very close to the aperture, and $\bar{v} \approx v_{0}$. If $E_{Z}(0, z)$ is known near the aperture, $\bar{v}$ can be calculated. In SPEAM, " it is assumed that the energy of the beam can be approximated by a cubic between $z_{1}=0$ and $z_{2}=3 r_{a}\left(r_{a}=\right.$ aperture radius), with the plane of the aperture at $z_{a}=2 r_{a}$ (shown in Fig. 2):

$$
w(z)=w_{0}+\left(\frac{z}{3 r_{a}}\right)^{3} r_{a} \Delta w / l
$$

The velocity of a particle of mass $m$ having a kinetic energy $W$ is

$$
v(W)=\sqrt{\frac{2 W}{m}} .
$$

*Note that the code makes allowance for electric fields to exist "outside the column" as in the case of multielectrodes (simulated by contiguous columns in TRACE); however, the formulation best models those conditions where there is either no field outside the coiumn or where there is a significant difference in fields between adjacent columns. 
The average energy near the entrance aperture is

$$
W_{a}=\frac{1}{3 r_{a}} \int_{0}^{3 r}\left[w_{0}+\left(\frac{z}{3 r_{a}}\right)^{3} r_{a} \Delta W / l\right] d z=w_{0}+0.25 r_{a} \Delta w / l .
$$

Assuming that $\bar{v} \approx v\left(\bar{w}_{a}\right)$,

$$
\bar{v}=\sqrt{\frac{2}{m}\left(\bar{w}_{a}\right)}
$$

Then,

$$
\Delta x^{\prime}=\frac{-\Delta W / \ell}{2 m v_{0} \sqrt{\frac{2}{m}\left(W_{a}\right)}} x=\frac{-\Delta W / l}{4\left(W_{0}\right)^{1 / 2}\left(W_{a}\right)^{1 / 2}} x,
$$

where $W_{0}$ is the energy at the entrance to the column. Treated as a thin lens, the entrance aperture then has a focal length equal to

$$
\frac{1}{f_{\text {ent }}}=\frac{-\Delta W / \ell}{4\left(w_{0}\right)^{1 / 2}\left(w_{0}+0.25 r_{a} \Delta W / l\right)^{T / 2}} \text {. }
$$

A similar exercise at the exit aperture results in an exit focal length equal to

$$
\frac{1}{f_{\text {exit }}}=\frac{\Delta W / \ell}{4\left(W_{f}\right)^{1 / 2}\left(W_{f}-0.25 r_{a} \Delta W / l\right)^{T / 2}},
$$

where $W_{f}$ equals $W_{0}+\Delta W$, the kinetic energy at the exit of the column.

Similar results are obtained when the cubic function is substituted into Eq. (4.31) in El-Kareh and EI-Kareh, ${ }^{8}$ a derived expression for the image focal length of an aperture lens:

$$
\frac{1}{f_{i}}=\frac{1}{4\left(\phi_{i}\right)^{1 / 2}} \int_{z_{0}}^{z_{i}} \frac{\phi^{\prime \prime}(z)}{[\phi(z)]^{1 / 2}} d z
$$

Note that the validity of the above formulations begins to break down as the ratio of $r_{a} / l$ increases above 0.25 . 


\section{APPENDIX C}

\section{FIELD FORMULAE FOR PERMANENT-MAGNET QUADRUPOLE}

The PMQ field formulae are obtained from Klaus Halbach, Ref. 5, to which we refer the reader for a further discussion of them and the assumptions under which they are valid. The formulae are based on the REC quadrupole of the standard design using trapezoidal blocks as shown in Fig. C-1 and are derived using a semi-infinite model with a flat-cut end (Fig. $(-2)$.

The fringe field to first order is as follows:

$$
\begin{aligned}
B_{x}(x, y, z) & =G(z) y=G_{0} F(z) y, \\
B_{y}(x, y, z) & =G(z) x=G_{0} F(z) x, \text { and } \\
B_{z} & =0
\end{aligned}
$$

where the gradient $G(z)$ is expressed in terms of the peak value $G_{0}$ times the fringe-field function $F(z)$ :

$$
G(z)=G_{0} F(z),
$$

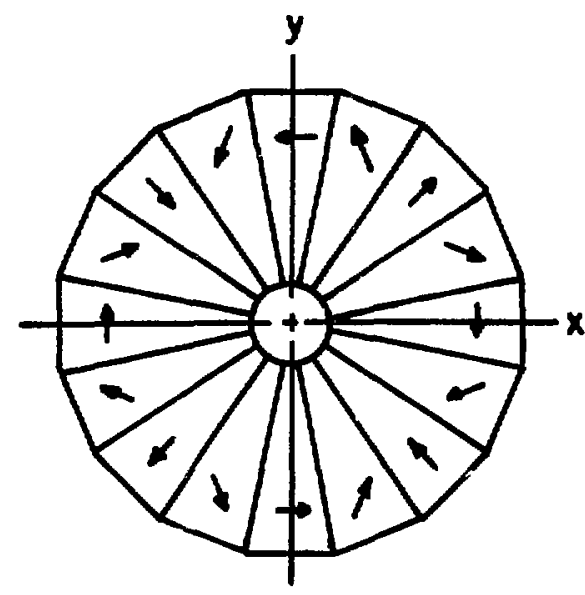

Fig. $C-1$

Cross section of segmented REC quadrupole (beam perpendicular to drawing plane).
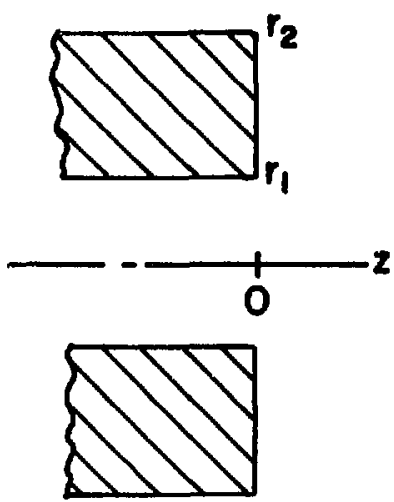

Fig. $\mathrm{C}-2$

Cross section through semi-infinite REC quadrupole (beam in drawing plane). 
and where the fringe field function $F(z)$, as shown in Fig. $C-3$, is given by

$$
F(z)=\frac{1}{2}\left\{1-\frac{z}{8}\left(\frac{1}{r_{1}}+\frac{1}{r_{2}}\right)\left[\frac{v_{1}^{2} v_{2}^{2}\left(v_{1}^{2}+v_{1} v_{2}+v_{2}^{2}+4+8 / \nu_{1} v_{2}\right)}{v_{1}+v_{2}}\right]\right\},
$$

where

$$
\begin{aligned}
& v_{i}=\left[1+\left(\frac{z}{r_{i}}\right)^{2}\right]^{-1 / 2}, i=1,2, \\
& G_{0}=2 B_{r} C_{2}\left(1 / r_{1}-1 / r_{2}\right)=2 G(0)=G(-\infty), \\
& B_{r}=\text { magnitude of remnant magnetization of REC mat orial, } \\
& r_{1}=\text { inner radius of quadrupole, } \\
& r_{2}=\text { outer radius of quadrupole, } \\
& C_{2}=\text { sin }(3 \pi / M) /(3 \pi / M), \text { and } \\
& M=\text { number of trapezoidal blocks composing the magnet. }
\end{aligned}
$$

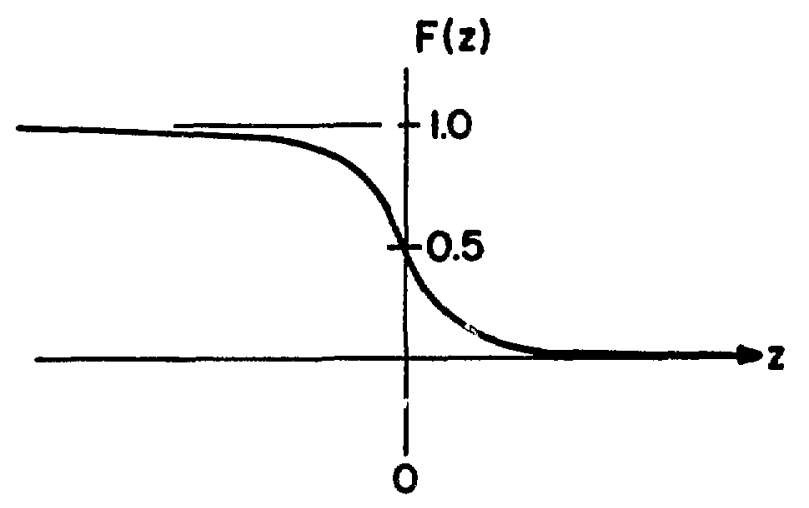

Fig. $\mathrm{C}-3$

Quadrupole fringe-field function. Note that $F(-z)=1-F(z)$. 
To find the fringe field for a quadrupole of finite physical length $\ell_{p}$, a second semi-infinite magnet of opposite sign is superimposed on the first semi-infinite magnet with a nonoverlapping distance equal to $l_{p}$. The fields are added, resulting in the fringe field as shown in the lower portion of Fig. C-4.

For doublets, triplets, or any comb ination of PMQs, the total fringe field $G_{t}$ is found by summing the individual gradients $G(z)$ as follows:

$$
G_{t}=\sum_{i=1}^{n} G_{i}\left(z_{i}\right)=G_{0 i} F_{i}\left(z_{i}\right), i=1, n
$$

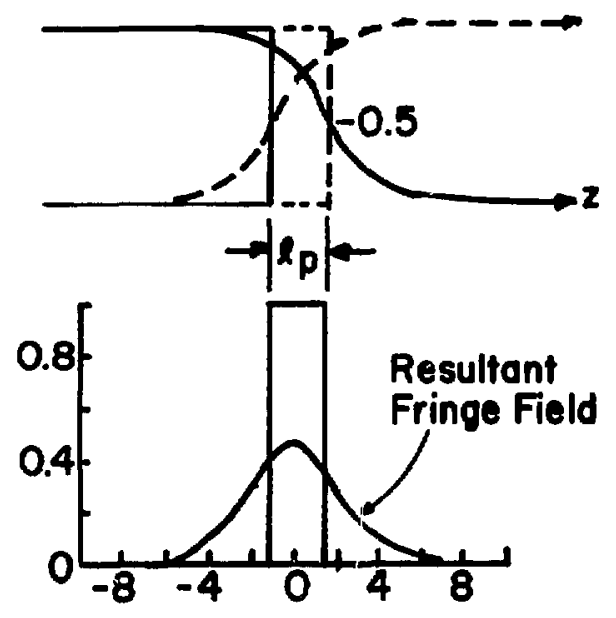

Fig. $C-4$

Two semi-inf inite magnets, nonoverlapping by $l_{p}$, resulting in fringe field for $P M Q$ of length $l_{p}$. 


\section{APPENDIX D}

\section{SPACE-CHARGE CALCULATIONS}

\section{SPACE-CHARGE CONTRIBUTION TO DEFOCUSING}

For a uniformly charged, continuous beam with an elliptical cross section having semiaxes $r_{x}$ and $r_{y}$ in the horizontal and vertical directions, respectively, the electric field that is due to space charge is ${ }^{9}$

$$
E_{x}=\frac{1}{4 \pi \varepsilon_{0}} \frac{4 I}{v} \frac{1}{r_{x}\left(r_{x}+r_{y}\right)} x
$$

and

$$
E_{y}=\frac{1}{4 \pi \varepsilon_{0}} \frac{4 I}{v} \frac{1}{r_{y}\left(r_{x}+r_{y}\right)^{y}},
$$

where $I$ is the beam current, $v$ the velocity of the particles, and $\varepsilon_{0}$ the permittivity of free space (mks units are used).

At higher velocities, the magnet ic force becomes important and acts in the opposite direction, thereby reducing the total defocusing force caused by space charge. The reduction factor is

$$
1-B^{2}=1 / \gamma^{2},
$$

where

$$
B=v / C \text {. }
$$

Space-charge forces give rise to the equations of motion:

$$
\frac{d}{d t}\left(\gamma m_{0} x\right)=a \frac{E_{x}}{\gamma^{2}} \text {, }
$$

and

$$
\frac{d}{d t}\left(\gamma m_{0} y\right)=q \frac{E_{y}}{\gamma^{2}}
$$

where $\gamma$ is the ratio of the total mass to the rest mass, $m_{0}$ is the rest mass, and $a$ is the charge on a single particle. When these differential equations in time are converted to differential equations in central-path length, using 


$$
v \frac{d}{d z}=\frac{d}{d t}
$$

the equations of motion become

$$
x^{\prime \prime}=a E_{x} / \gamma^{3} m_{0} v^{2}=k_{x}^{2} x
$$

and

$$
y^{\prime \prime}=q E_{y} / \gamma^{3} m_{0} v^{2}=k_{y}^{2} y .
$$

The $k_{x}{ }^{2}$ and $k_{y}{ }^{2}$ factors in these equations are not constants, however, but depend on the beam profiles, $r_{x}$ and $r_{y}$, which, in turn, depend on the path length $z$. In the calculations, the space-charge contributions to $k_{x}{ }^{2}$ and $k_{y}{ }^{2}$ are assumed to be constant during each step, but an iterative procedure is applied to ensure that good average values are used.

The original estimates of the space-charge forces $k_{x}{ }^{2}$ and $k_{y}{ }^{2}$ are taken to be these quantities evaluated at the beginning of the step. After the beam has been followed through this calculational step, the program has an estimate of the beam profile at the end of the step. As a matter of fact, $k_{x}{ }^{2}$ and $k_{y}{ }^{2}$ and their first derivatives can be evaluated both at the beginning and end of the step. These four quantities are used to specify the coefficients of a cubic polynomial for approximating $k_{x}{ }^{2}$ and $k_{y}{ }^{2}$ over the step interval. These cubics are used to determine improved averages of $k_{x}{ }^{2}$ and $k_{y}{ }^{2}$ over the step length. This procedure is iterated twice.

It is important to remember that the model in TRACE is for a uniform charge distribution in $x, y$ space over an ellipse with semiaxes $r_{x}$ and $r_{y}$ in the horizontal and vertical dimensions. In addition, it is a two-dimensional model and, hence, neglects effects from the finite length of a micropulse. Furthermore, the debunching of short bunches that is due to momentum spread and space-charge forces is not included. The values for $r_{x}$ and $r_{y}$ are taken from the beam matrix. In the TRACE model, a 11 of the beam is in the ellipse described by the beam matrix. 


\section{APPENDIX E}

\section{SAMPLE OUTPUT}

This appendix includes six figures, four of which show the graphic output from typical TRACE runs. Figure E-l illustrates a set of calculations (variation of space charge and the resultant mismatch) and how they are recorded automatically down the left-hand side of the page. Figure E-2 shows a beamline that includes most of the elements available in TRACE. In Fig. E-3, we see two different measured beams compared on the same graph, using the "Y" command to identify the different envelopes by date of measurement. Figure E-4 is a copy of the data file printout using the "p" command. The output in Fig. E-5 is the result of an RFQ radial match using the "Q" cormand with an S-shaped radial match section at the RFQ entrance. Finally, Fig. E-6 depicts a typical TRACE ASCII data file as seen by an editor. 
F ILE CONWENT ?

EEAM MEASURED ON AT EMITTANCE STATION \&2

NOTE : AT EMD OF BEAMI.INE:

IIGMA (BEAM) MATRIX ELEMENTS

SoRT SIG(1.1). 1.48606 as

SORT SIG(2, 2): $55.16161 \mathrm{mr}$

sokr sict3.3)

SORT SIG(4,4) 24.38032 -

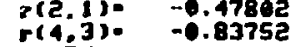

$\begin{array}{rr}0.673 & -0.032 \\ -18.015 & 1.512\end{array}$

-9.688 R. -2.079

MOTE: UARY SPACE CHARGE WND SEE

EFFECT ON nISAATCH

ENTER IMPUT ( . . . ...

XI=3

HOAI2 AISHATCH2 2,35

UEAT. MISHATCHIEZ 650

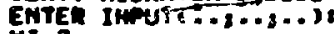

XI-2.

thent 1.200

Exte: Mimpite

xI-:

LERT MIsFATCH: 0.095

ENTË IMPUT $(. ., \ldots 3 .$.

$\times 1 \cdot .5$

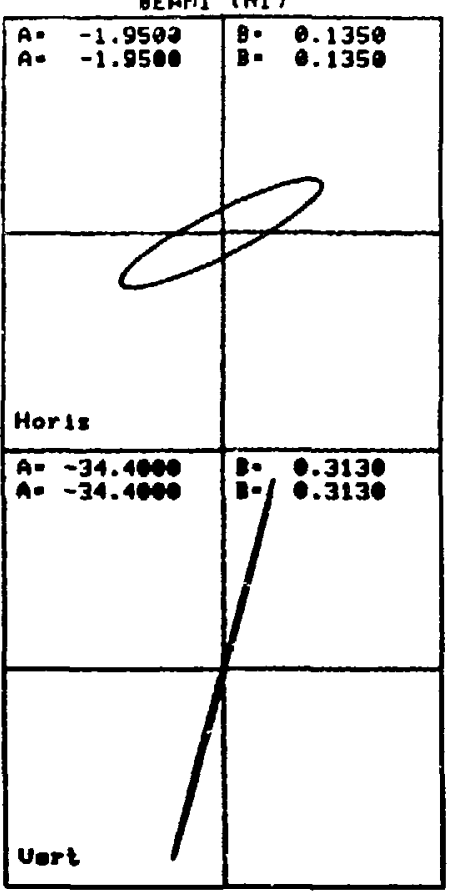

$\begin{array}{lrr}\text { I. } & 1.0 & 5.0 \\ \text { U. } & 0.020 & 0.109 \\ \text { EX. } & 161.00 & 72.00 \\ \text { EY. } 18.00 & 8.05 \\ \text { MATCHING } & \text { UARIALLES } \\ \text { ME } & \text { MP } & \text { UALUE } \\ 23 & 1 & 1.474 \\ 25 & 1 & -2.997 \\ 27 & 1 & 3.763 \\ 29 & 1 & 0.060\end{array}$

MATCHIMG TYPE - I

DESIRED UALUES?

ALPHA-X 0.54

ALTAW..

ALPHA-Y 8.64

MISHATCH FACTORS:

X-plene 0.096

PRINTOUT UALUES

PE PP UALUE

18 1 $12.5 \%$

20 1 - $12.5 \mathrm{eb}$

$21 \quad 1 \quad 262.269$

ouns, poExt

COpE: TRACE ul

DATE: 14-JUN-34

TIRE 14255805

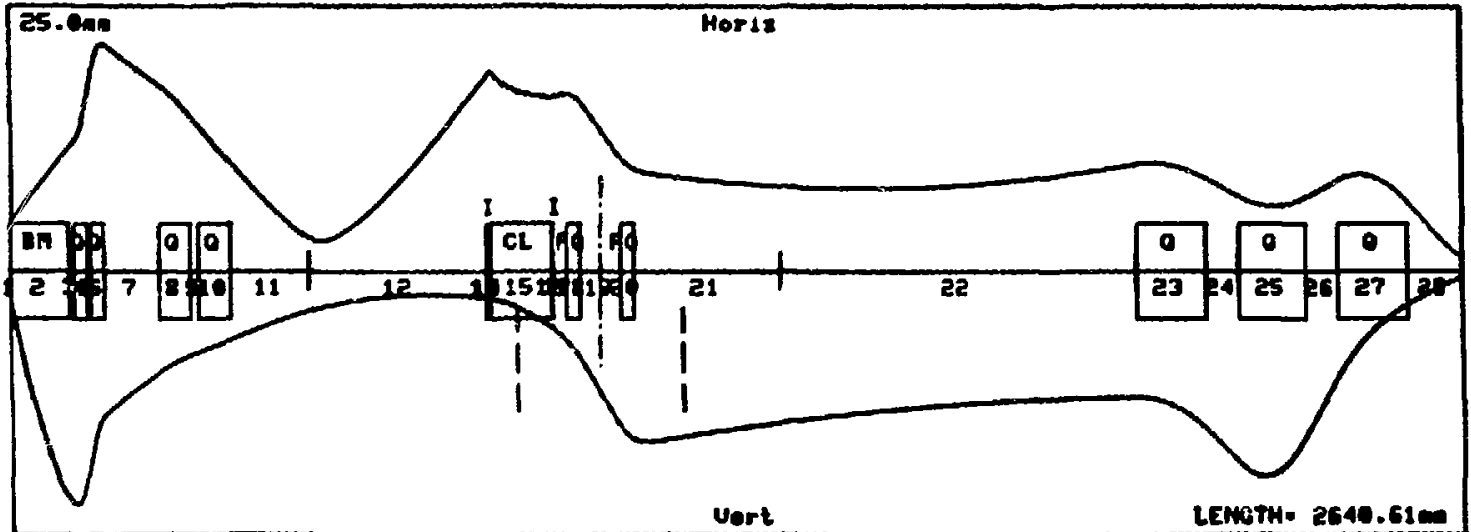

ENTER IFPTT ... $\mathrm{x} 1=1$

nOTE\& cET MATRIX AT COLUNH EXIT EMTER INPUT(........

$$
\text { Na.15 }
$$

N1* 1 N2-15 SIGM (IEAT) MATRIX ELENENTS sont sig(1,1): 16.41877 sont sig(z,z) 25.86545 Sont SIG(4,4): 19.46243 ar r(e, 2$) 0$ -. 9552 $+(4,3) 0$

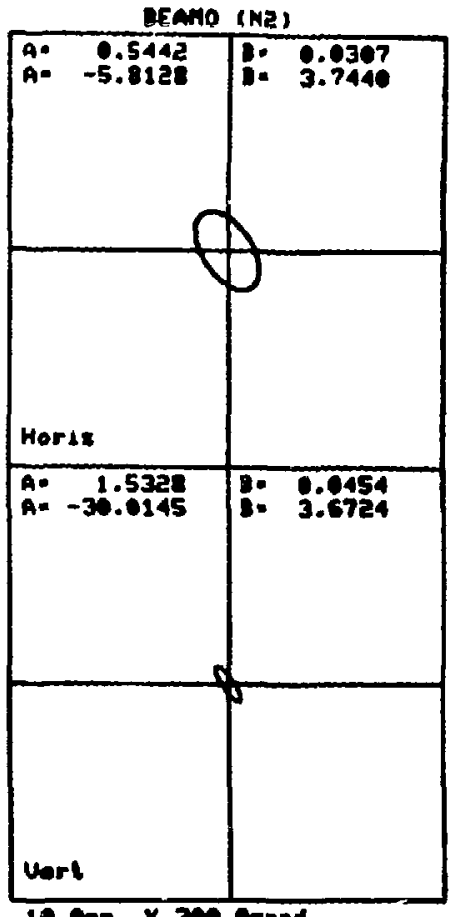

10.600 $\times$ 300.6arad

Fig. E-1. Output graphics. 


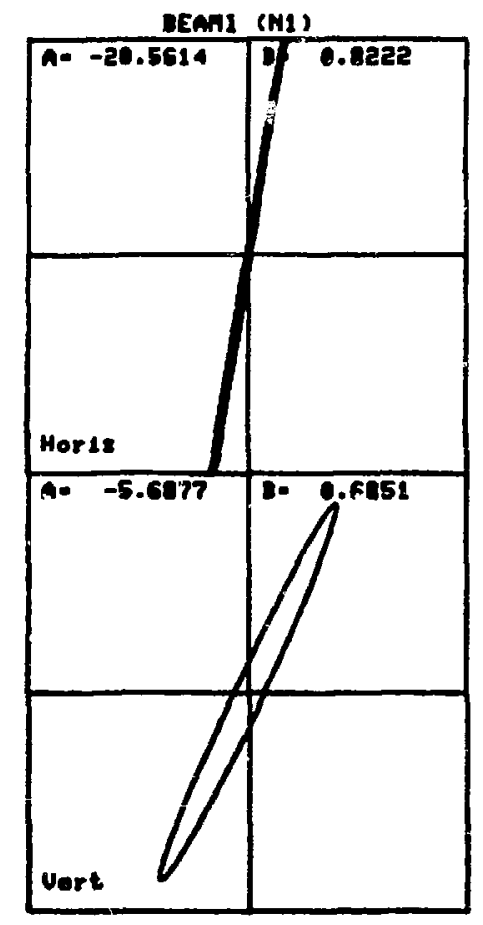

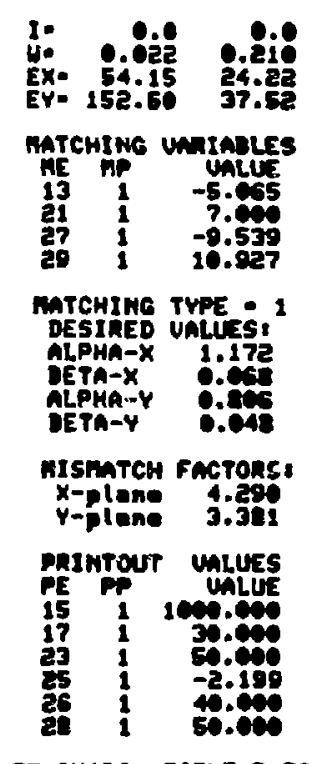

m cuabs, pesxt-2.50

CODEI T2OCE 01

FIIE: MINESTROW. DAT: parte? 14-JUN-24.
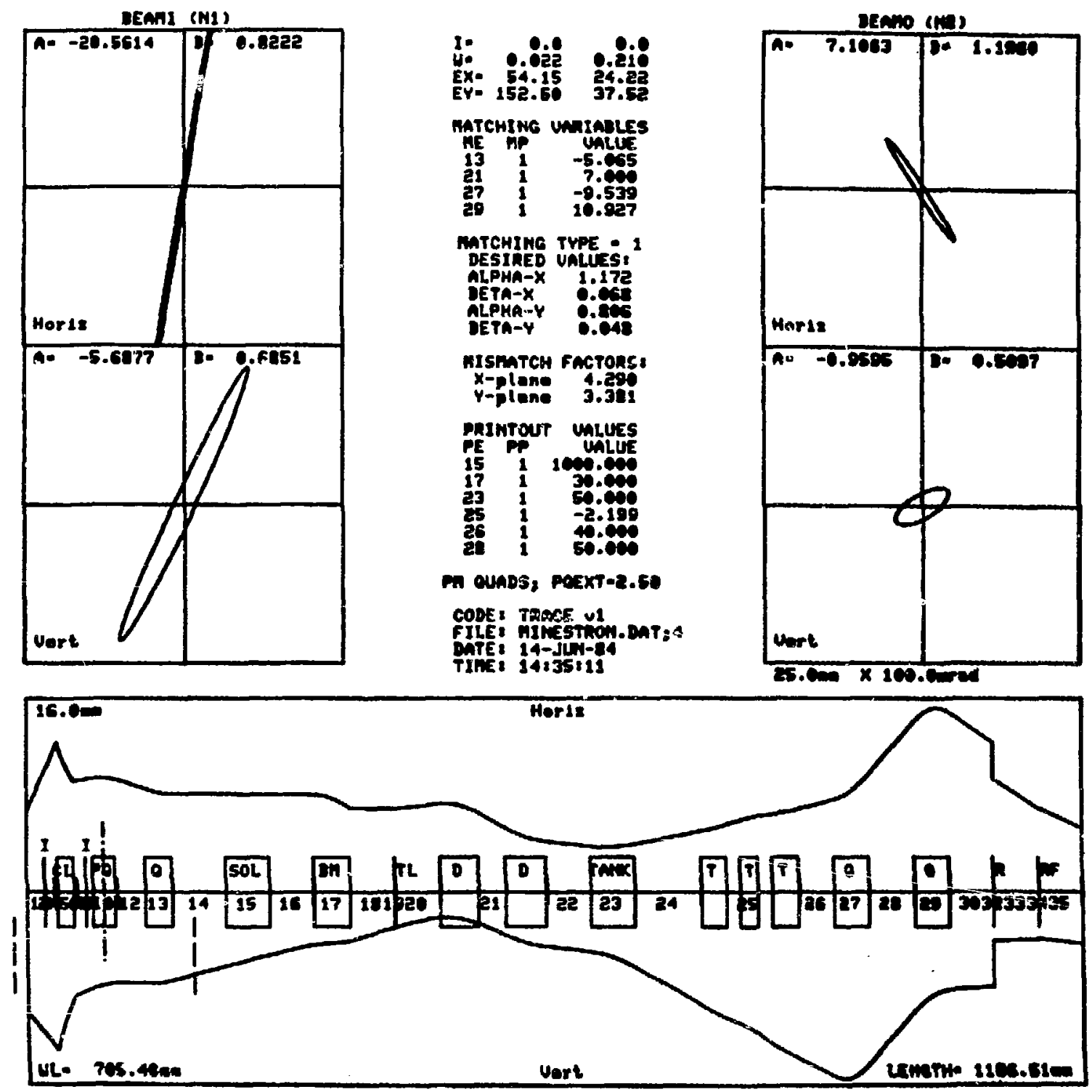

Fig. E-2. Output graphics. 
DEANI (NI)

- ILF COMNENT: PACK TRACED - CURRENT-106I

MOTE: ENTER MEU SEANI NEASURED CMTER 31 - HAY -84

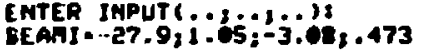

HOR12 MISNATCH: 2.955

UERT. MISHATCH: 1.208

8.16 116.74 11.66 7.39

COUPLE TO FOLLOANG PARANETERS

G6.74 55.76 51.18 12.21

MTchimg-staupey,

(COUPLED UARIALLES)

INIT. MH - 2.955

F Twi 1.358

MATCHING-STAMDUY: (HIT* 10)

(COUPLED UARIAULES)

INIT_ n: 1.35

TMn m - 1.193

matCHING-STanjeys (NIT- 10)

COUPLED UARTALLES

IMIT $\mathrm{m}$ : 1.193

MOAI2 nishatch: 1.17

MEnT. MISHATCH: 1.173

MATCHIM VARIADLE UALES:

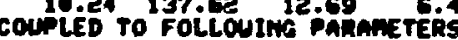
$-A(1,12)-A(1,16)-A(1,24)-A(1.32)$

DEAMLINE LEMGTH: G72.73m

MOTEI F IELDS OUTSTDE EXTEMD FAMTHER PHAN SHOW

Uart
1. $\quad 0.00 \quad 69.0$ EY. $231.16 \quad 28.69$

MATCHIITG UARIAILES

ME TP UALUE

$\begin{array}{rrr}8 & 2 & 8.164 \\ 13 & 1 & 116.743\end{array}$

$\begin{array}{lll}137 & 1 & 11.659 \\ 25 & 1 & 7.394\end{array}$

MATCHING TYPE - 1

DESIRED VALUES:

ALPHA-X

DETA-X.

ALPHA-Y

1.172
-.195

.806

misnatCh FACTORS!

$x-p l a n e$
$y-p l a n e$
0.512

4. -3.8573 :- 1.3931

PRINTOUT UALUES

PE PP UALUE

$\begin{array}{lll}12 & 1 & 65.736 \\ 15 & 1 & 55.760\end{array}$

$24 \quad 1 \quad 51.184$

331319.65

m ounds, PoExT-2.50

CODE: TRACE vt

FILE! 3MAMYA4.DAT B

DATE: 14-JuN-p4

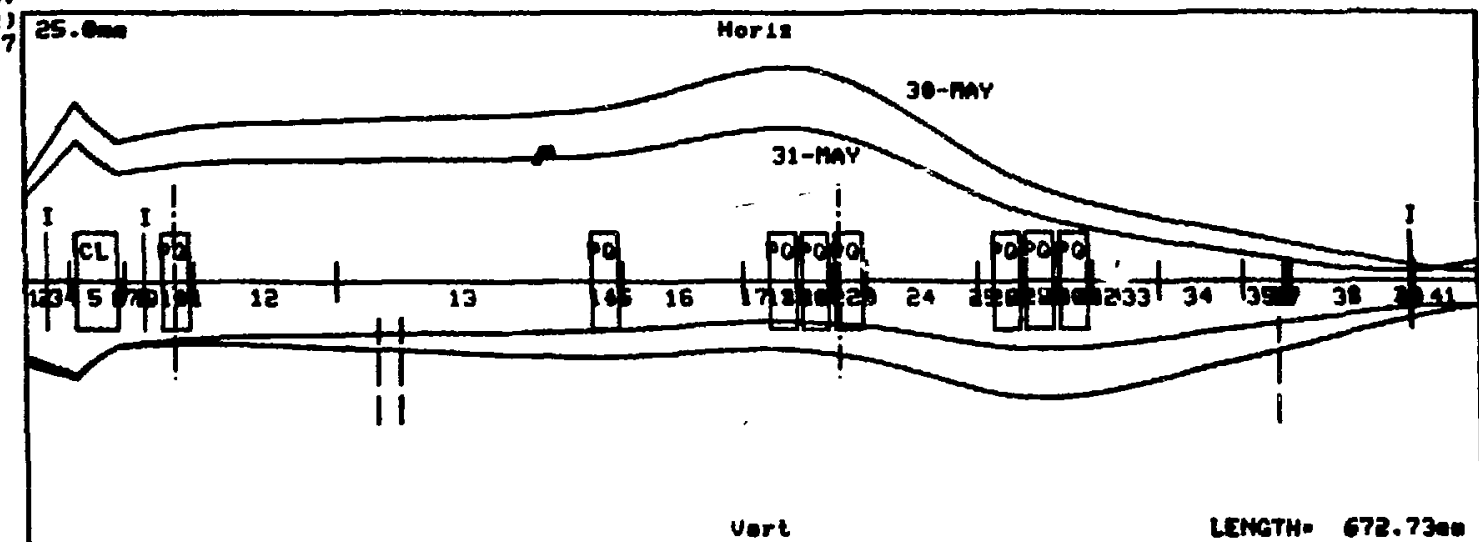

DEAHO (NE)

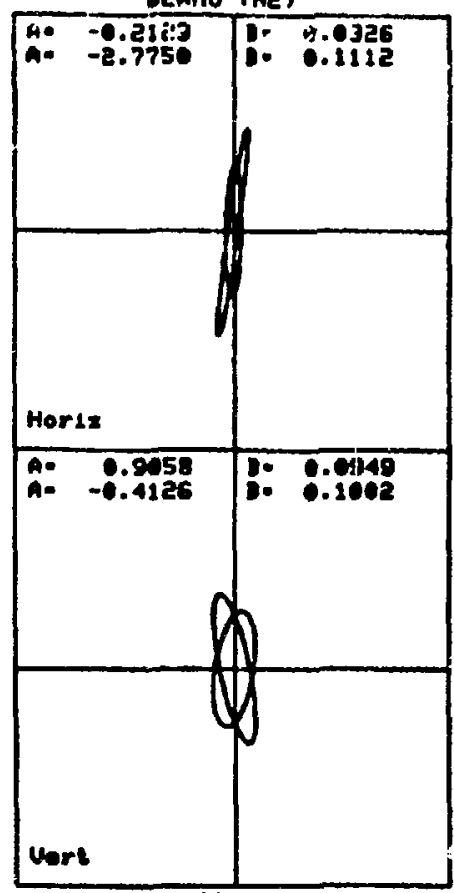

. On $\times$ 14e.cerad

Fig. E-3. Output graphics. 
ENTER FILEMAME OR HIT AETURN FOR DUMAY IMPU

IHPUT F ILEMME: ?MARSA

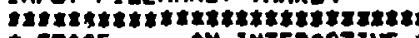

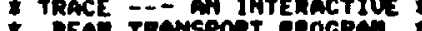
- Tor reto Tupe 14-JUN-24 14126:49 FIEMAne: 7mande.0AT, 1

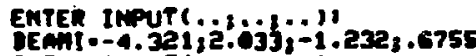

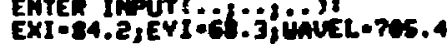

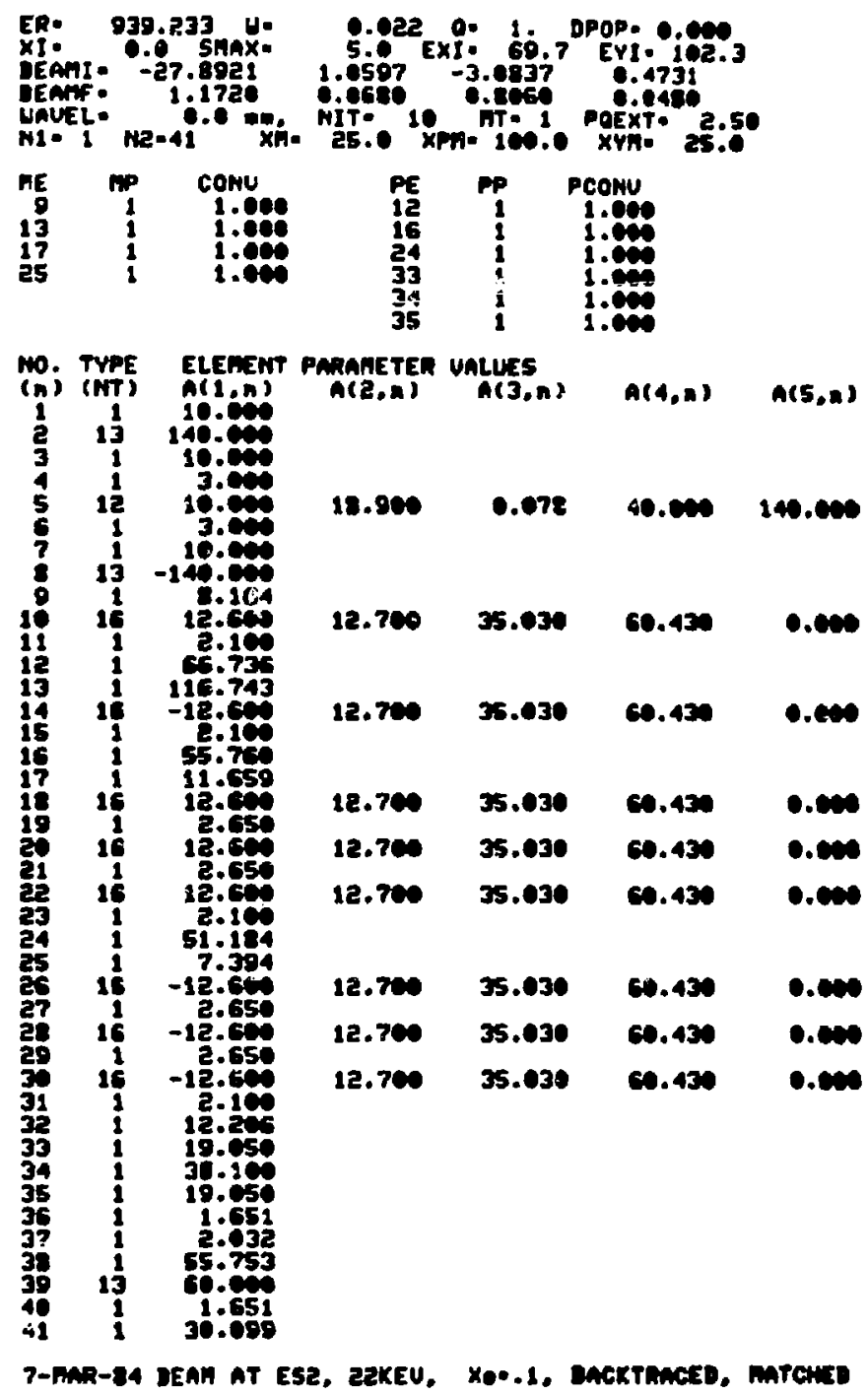

Fig. E-4. Data file printout using the "P" command. 


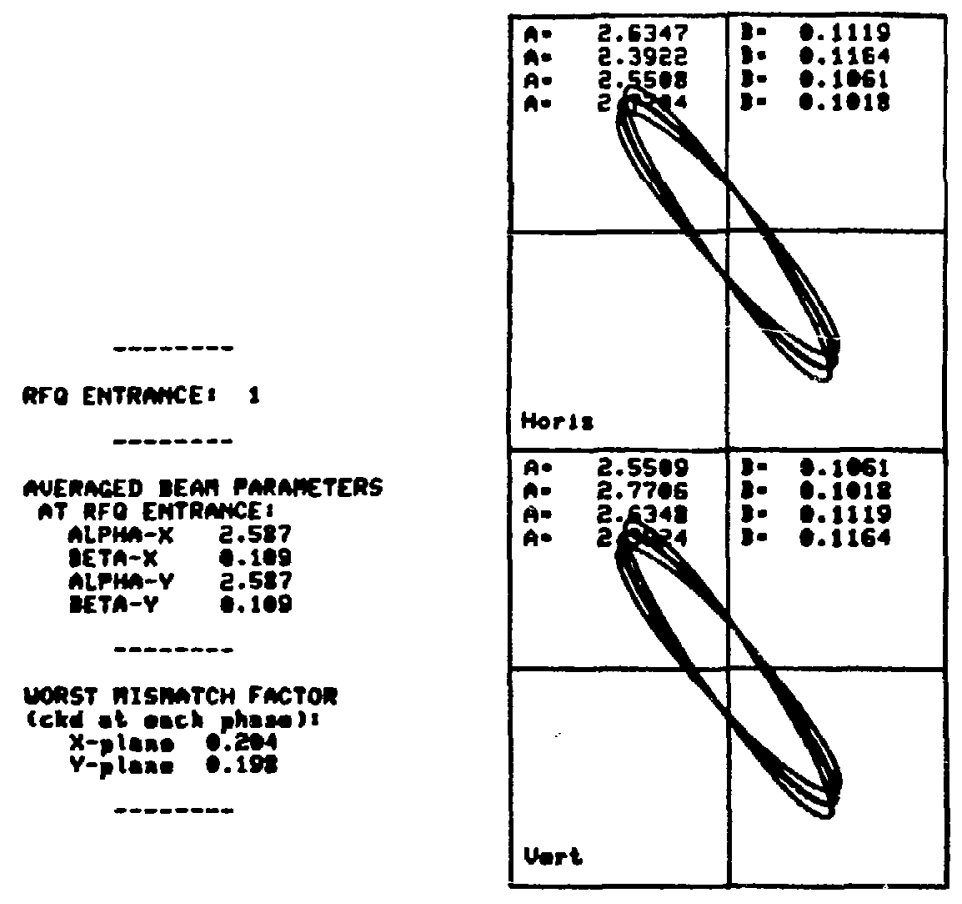

$\begin{array}{lll}\text { I. } & 150.0 & 150 . \\ \text { EX. } & 55.00 & 0.10 \\ \text { EY. } 55.00 & 55.0\end{array}$

MATCHING UARIADLES

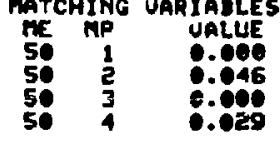

MATCHING TYPE : 6

DESIRED UALUES:

(INAPUT DEAN UALUES)

ALPHA-X 0.000

BETA-X

ALPHA-Y

PRIMTOUT UALUES

PE PP UALUE

CODE: TRACE WI

FILE: RFO.DAT 3

DATE: 19-JUN-C4

EFo michine
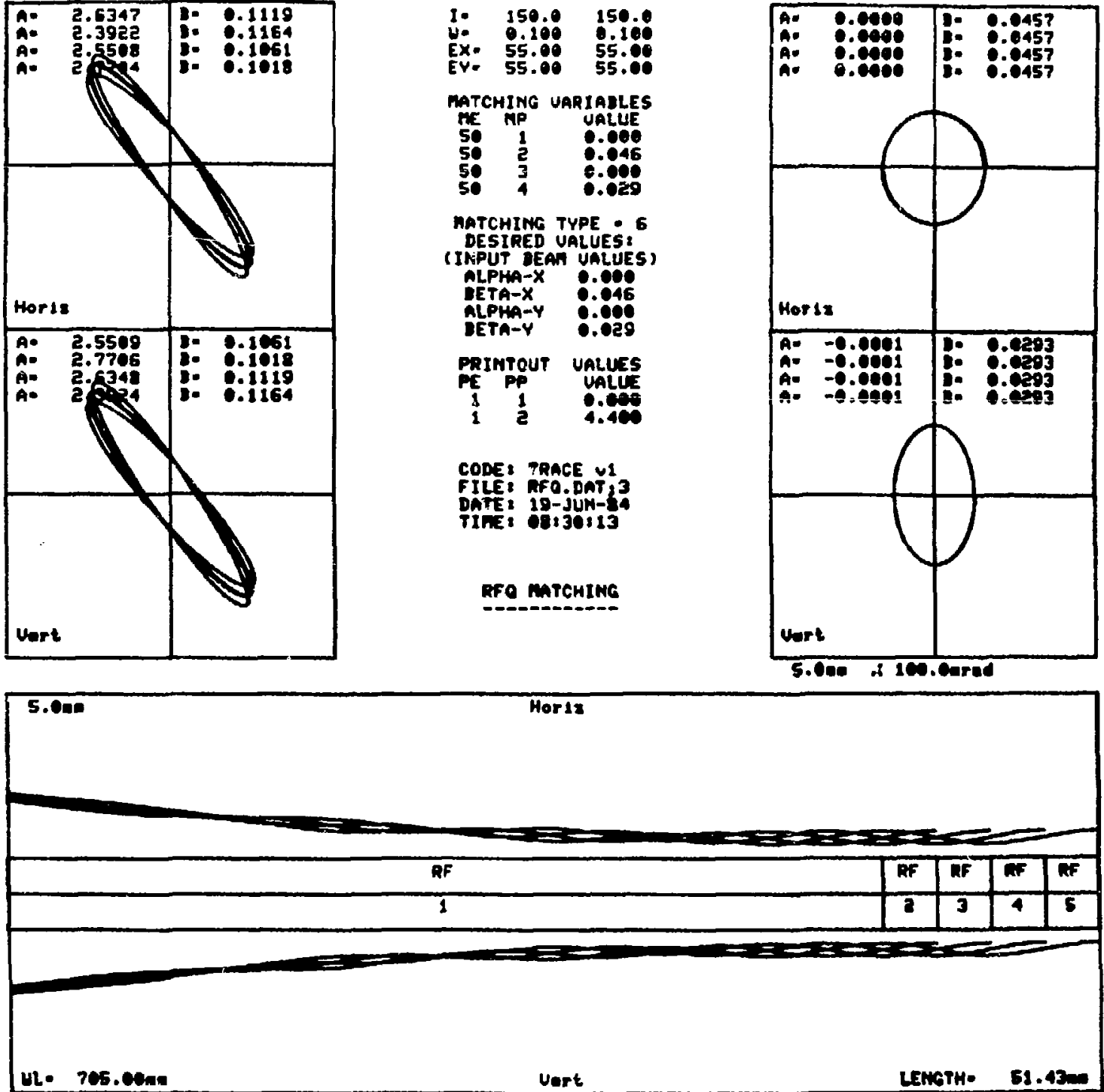

Fig. E-5. Output graphics of RFO radial match using " 0 " command. 

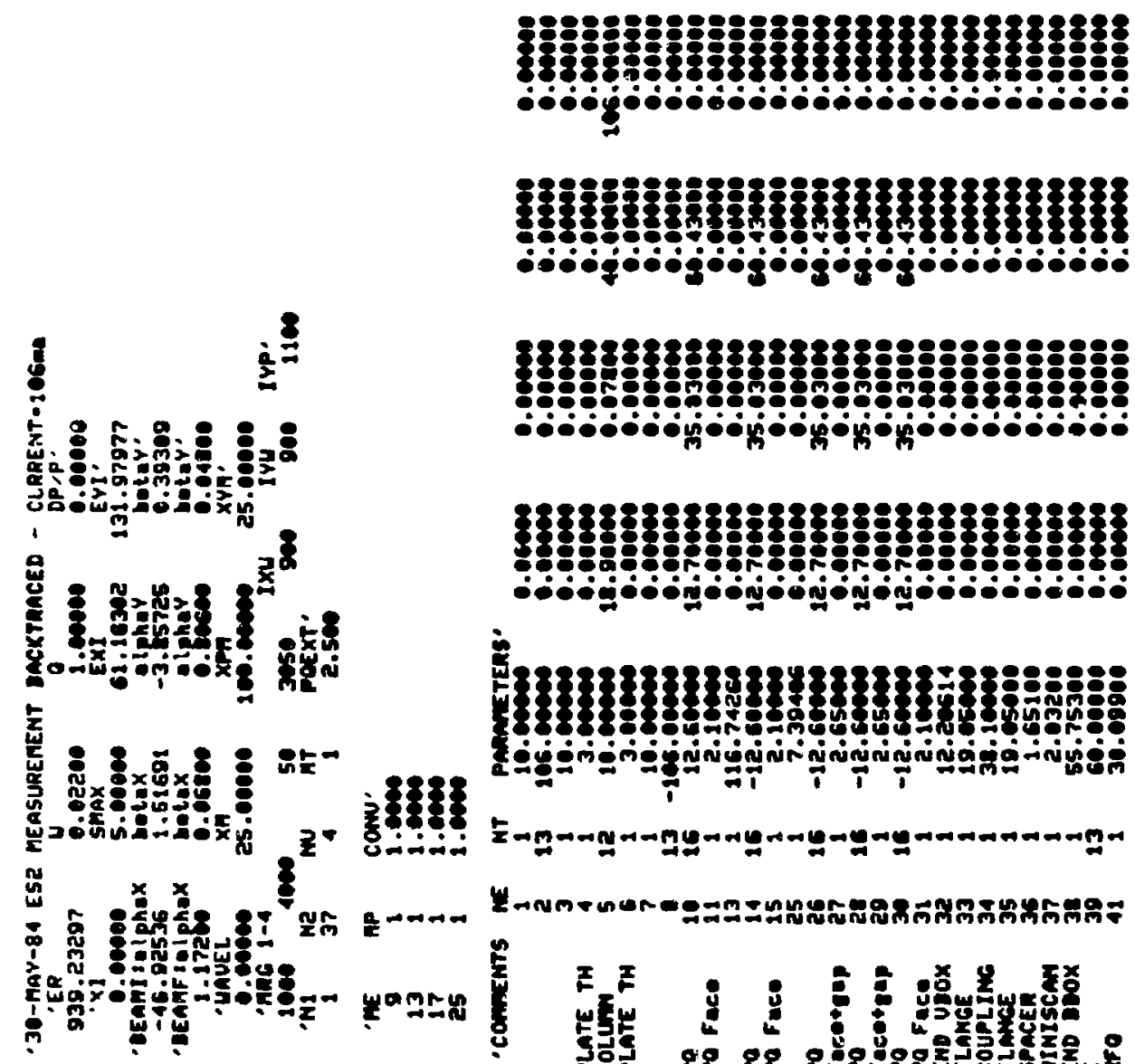

엉

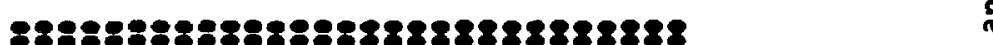

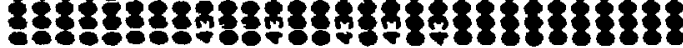

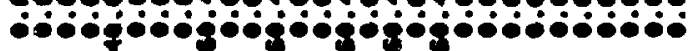

르

ष्.

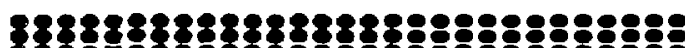

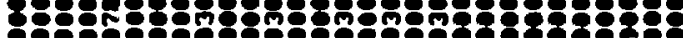

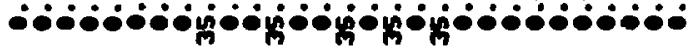

$\frac{a}{4}$

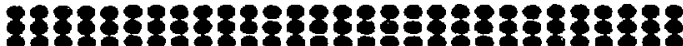

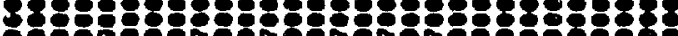

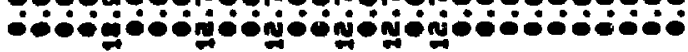
$\dot{8}$

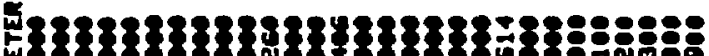
121820 to

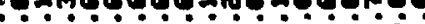

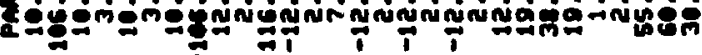

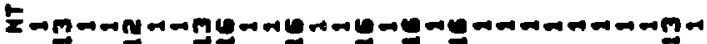

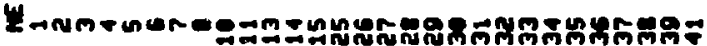
密
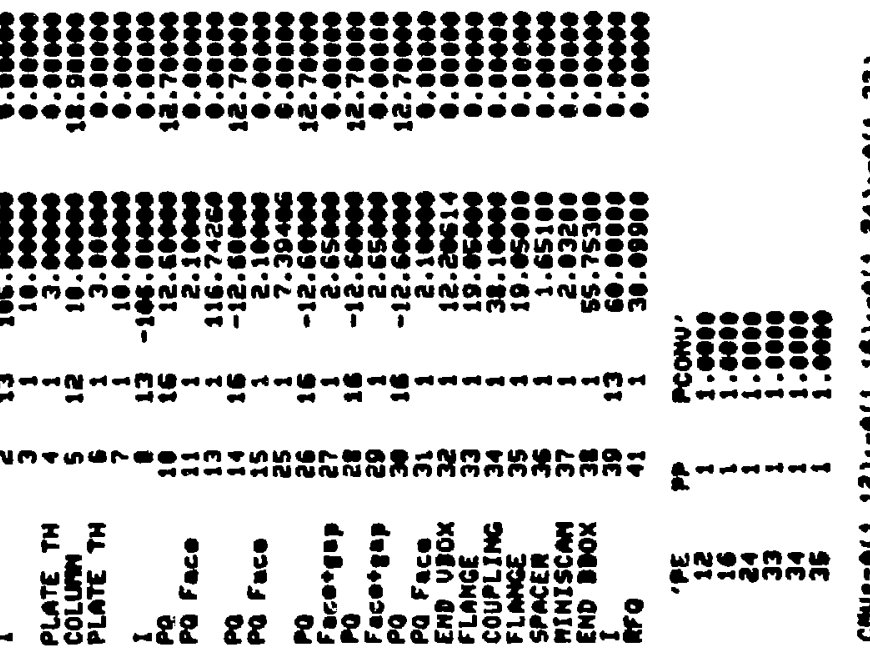


\section{APPENDIX $F$}

\section{HELP PACKAGE}

The three figures included in this appendix are the three pages of the on-line HELP package in TRACE. Figure $F-1$ is a printout of Page 1 , which lists the available commands, the transport element types, and the general input variables. Page 2, shown in Fig. F-2, identifies the parameters for each of the transport system elements. Page 3, reproduced in Fig. F-3, lists the types of matching possible and shows how to set up each matching procedure. The HELP package is accessed at any time from the wait-for-command mode by typing the letter "H." 


\section{INSTRUCTIONS FOR DATA IMPUT}

OATA IMPUT IS PROMPTED UITH THE HORDS 'ENTER INPUT: THE DATA IS ENTERED IH THE FOLLOUING FORH?

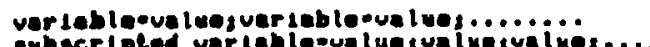

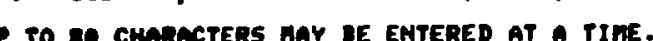

FIELDS TUST DE SEPAMATED UITH SENICOLOHSi, $)$.

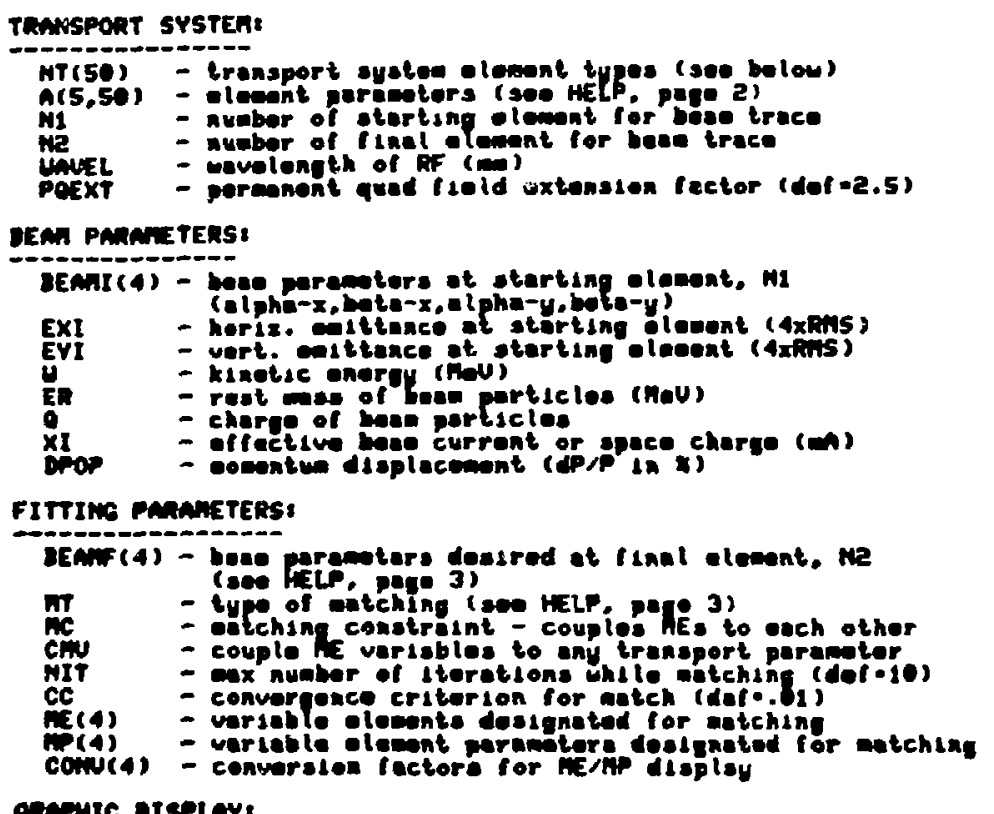

oxamic display,

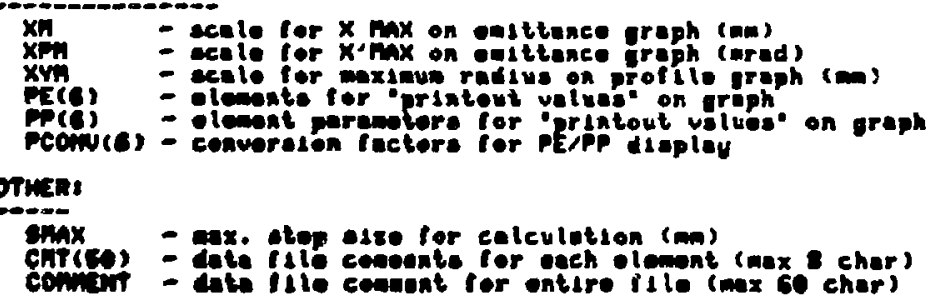

TO IMPLEMENT THE CORRESPONDING COMMAND, TYPE,

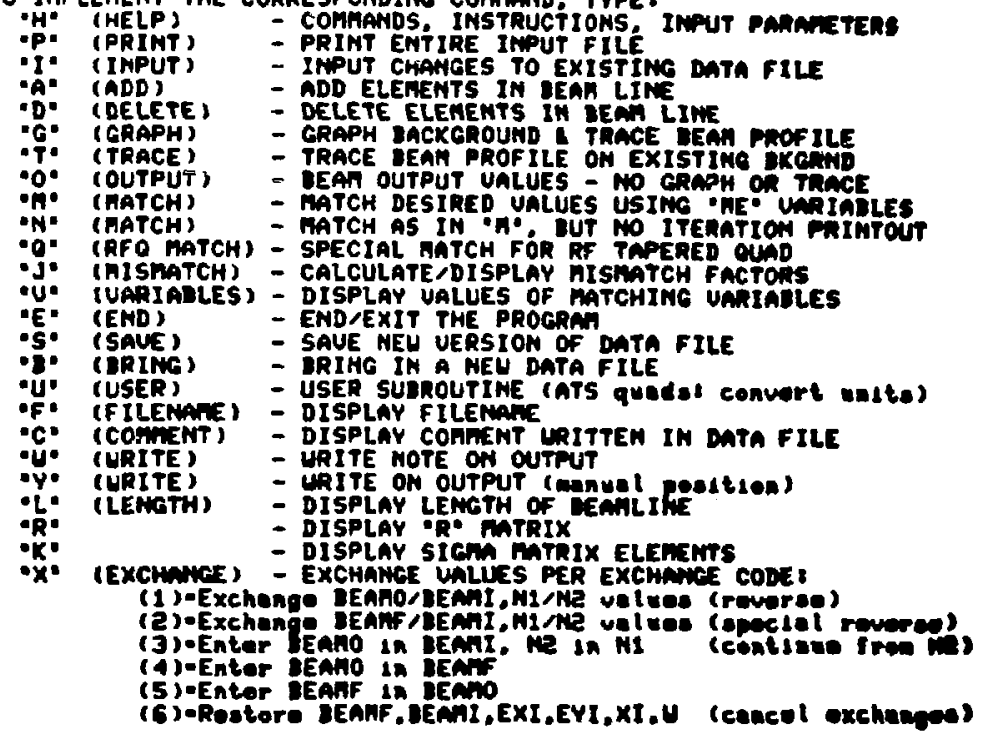

TRANSPONT SYSTEW - ELEWENT TYPES (IT)

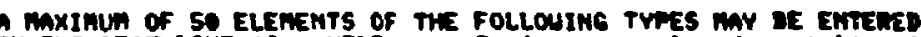
IN THE IEAN LINE (500 HELP page 2. (or poramotor doscriptions)

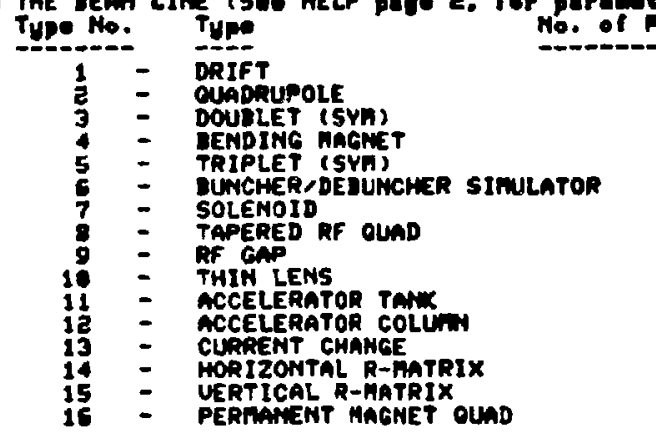

1
2
3
5
5
5
2
5
3
5
5
1
4
5

(Typo 'H* (or MELP, page 8 )

Fig. F-1. TRACE HELP package, page 1. 


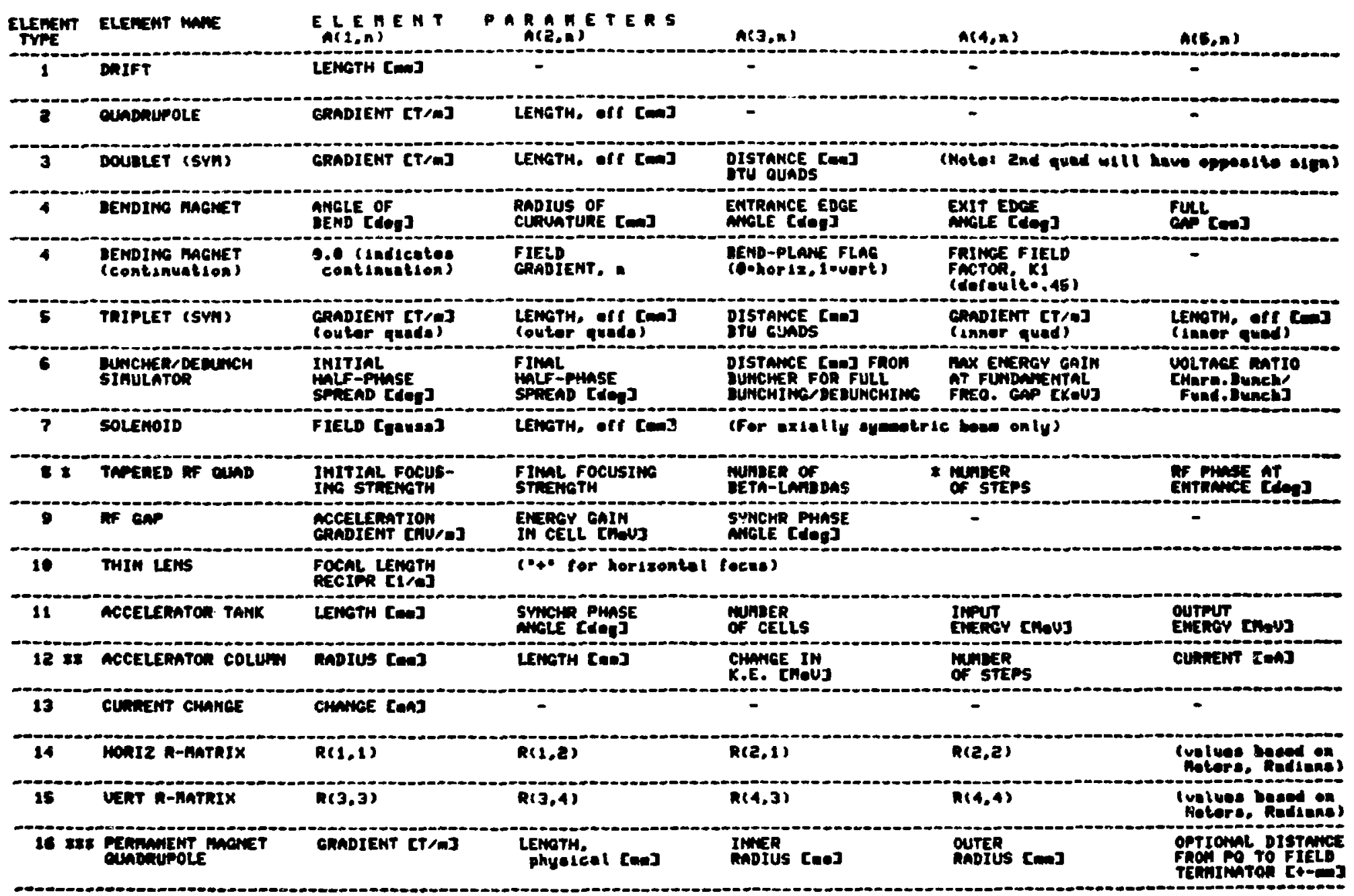

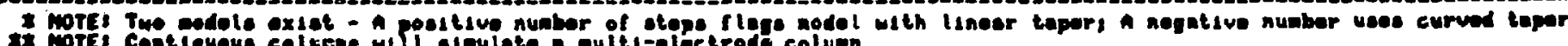

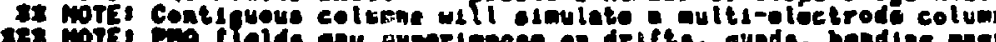


Trece HeLP. Mage 3, Ui

TYPES OF MTCKIKO

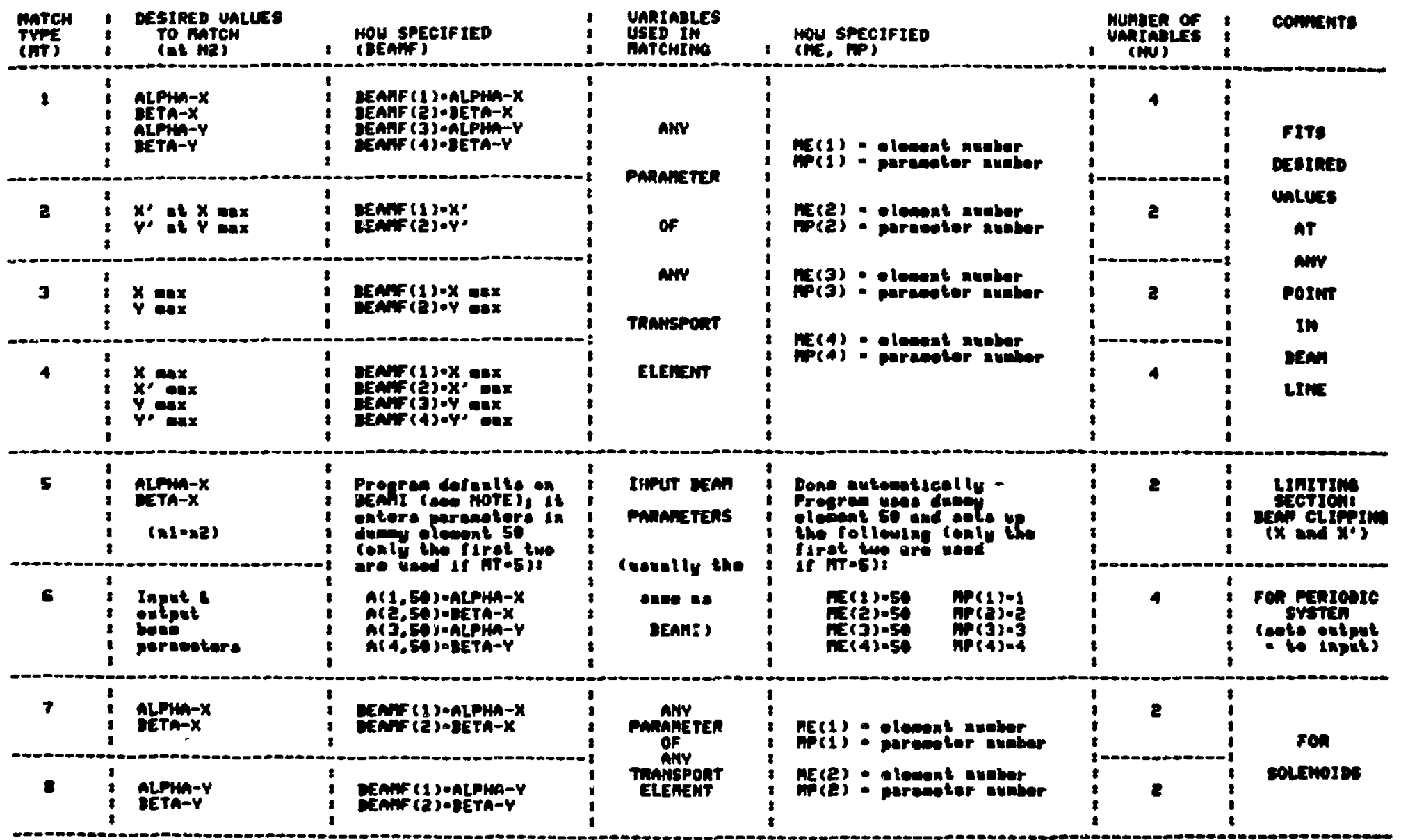

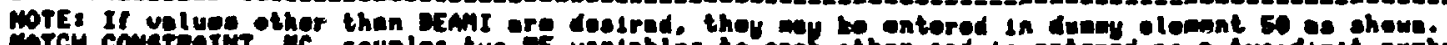

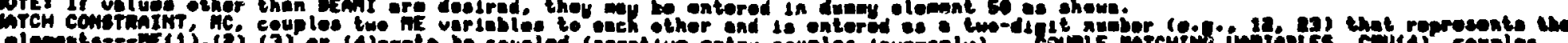

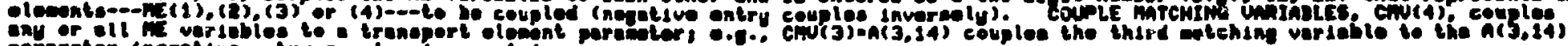

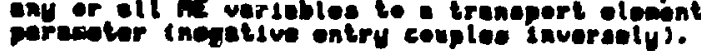

Fig. F-3. TRACE HELP package, page 3. 


\section{APPEMDIX \&}

\section{GRAPHICS PACKAGE}

The graphics in TRACE are generated by the Tektronix PLOTIO Terminal Control System-a relatively small part of the Tektronix PLOTIO graphics package. The program is set up to plot on any of the 4000-series Tektronix terminals and includes only the following calls:

\begin{tabular}{|c|c|c|}
\hline Call & $\begin{array}{l}\text { No. of } \\
\text { Times } \\
\text { Used } \\
\end{array}$ & Purpose \\
\hline initt, term & 1,1 & initializes, identifies terminal \\
\hline chrsiz, csize & 1,1 & sets character size \\
\hline scursr & 1 & calls up cursor cross hairs \\
\hline tsend & 1 & dumps buffer (optional) \\
\hline erase, bell & 2,3 & erases screen, rings bell \\
\hline drwabs & 3 & draws solid lines \\
\hline dshabs & 3 & draws dashed lines \\
\hline aoutst & 230 & outputs character string \\
\hline anmode & 230 & switches to alpha-numeric mode \\
\hline movabs & 260 & moves cursor to desired location \\
\hline finitt & 1 & resets terminal at program exit \\
\hline
\end{tabular}

Essentially, PLOTI0 can be replaced by any simple graphics package that draws lines, outputs character strings, and controls cursor position. The CDC7600 version contains a few more of the "anmode" and "movabs" calls than listed above because of compiler differences. 


\section{REFERENCES}

1. K. R. Cranda11, "TRACE: An Interactive Beam-Transport Program," Los Alamos Sc ientific Laboratory report LA-5332 (October 1973).

2. K. R. Crandall, "TRACE: An Interactive Beam-Transport Program for Unbunched Beams," CERN/PS/LIN/Note 77-3 (February 1977), and "Addendum to Program TRACE."

3. F. Sacherer, "RMS Envelope Equation with Space Charge," CERN/SI/Internal Report 70-12.

4. W. Joho, "SPEAM, A Computer Program for Space Charge Beam Envelopes," TRIUMF, Design Note TRI-DN-73-11, March 1973.

5. K. Halbach, "Physical and Optical Properties of REC Magnets," Nucl. Instr. and Meth. 187 (1981), 109-117.

6. See CERN Internal Report CERN/MPS/LIN 74-1.

7. M. Weiss, "Bunching of Intense Proton Beams with Six-Uimensional Matching to the Linac Acceptance," CERN/MPS/LI 73-2.

8. A. B. E1-Kareh and J. C. J. E1-Kareh, Electron Beams, Lenses, and Optics, Vol. I, (Academic Press, New York and London, i970), p. 66.

9. M. Kapchinskij and V. V. Vladimirskij, Conference on High Energy Acceleration and Instrumentation, CERN, Geneva (1959), p. 274. 